\title{
Linear elastic chain with a hyper-pre-stress
}

\author{
M. Charlotte ${ }^{(1)}$ and L. Truskinovsky ${ }^{(2)}$ \\ (1) Laboratoire des Proprietes Mecaniques et Thermodynamiques des Materiaux (UPR CNRS 9001), \\ Universite Paris-Nord, 93430 Villetaneuse, France. \\ (2) Department of Aerospace Engineering and Mechanics, University of Minnesota, Minneapolis, MN55455, \\ USA.
}

\begin{abstract}
To account for surface relaxation in ultra-thin films, we consider the simplest one-dimensional discrete chain with harmonic interactions of up to second nearest neighbors. We assume that the springs, describing interactions of the nearest neighbors (NN) and next to nearest neighbors (NNN) have incompatible reference lengths, which introduce a hyper-pre-stress and results in a formation of the exponential surface boundary layers. For a finite body loaded by a system of (double) forces at the boundary, we explicitly find the displacement field and compute the energies of the inhomogeneous stressed and reference configurations. We then obtain a simple expression for the hyper-pre-stress related contribution to the surface energy and show an unusual scaling of the total energy with the film thickness. For ultra-thin films we report an anomalous stiffness increase due to the overlapping of the surface boundary layers. Implications of the micro level hyper-pre-stress in fracture mechanics and in the theory of non-Bravais lattices are also discussed.
\end{abstract}

Keywords: a) surface effects, fracture b) thin films c) discrete lattices

\section{Introduction}

The growing demands of modern industry require an understanding of the mechanical behavior of nano-meter size objects. An extreme miniaturization of the mechanical structures makes classical continuum models incomplete for their adequate description mostly because the associated size effects can no longer be neglected. As an example, one can mention new technologies utilizing ultra-small actuators and sensors, where a satisfactory account for the microscopic surface boundary layers is essential. Here we are using the term ultra-small to identify objects where the bulk and the surface contributions to the elastic energy can not be considered independently.

As a first step in the direction of understanding the size effect in ultra-thin mechanical structures, here we study a discrete model accounting for surface relaxation in a linear solid. The relaxation is localized in the areas adjacent to the unloaded free surfaces and is due to the presence of the broken bonds; the size of the corresponding layers is measured in the units of a single atomic cell. Conventional discrete models limited to the nearest neighbor (NN) interaction fail to capture these effects and we, following some previous work, employ a model with the simplest next to nearest neighbor (NNN) interaction.

The object we model can be viewed as an infinite crystalline plate. The relaxation of the atomic layers parallel to the surface produces a nontrivial inhomogeneous configuration: lattice spacing normal to the surface of the first few atomic layers differs 
from the spacing of the deep layers. The structural relaxation manifests itself through the diffuse x-ray diffraction and broadened phonon scattering profiles (e.g. [GMH61, HLS92]). To secure the presence of the boundary layers at the free surfaces, we assume that the effective springs, describing NN and NNN interactions have incompatible reference lengths which introduces a mismatch or pre-stress. As a result, the macroscopically unloaded crystal in equilibrium will not be free of micro-stresses generated by an effective system of self-equilibrated forces and self-equilibrated couples. In view of the fact that the corresponding couples are "invisible" at the macroscopic level, the resultant pre-stress does not fit the definition of a standard pre-stress of the classical continuum elasticity theory and we use the term hyper-pre-stress to distinguish the two. Our type of pre-stress plays an important role when the micro-scales are important, for example when the crack opens inside the solid and the associated relaxation layers appear in the areas adjacent to the newly formed free surfaces. For sufficiently closely located free surfaces, as in the case of an ultra-thin film or a tip of a crack, the relaxation strain fields will overlap, producing a nontrivial energy and effective stiffness dependence on the external length scale. The goal of this study is to make a simple quantitative model of these effects allowing one, for instance, to study the effective surface energy scaling with the size of the body.

Given that in our setting, the forces and displacements vary only in the direction normal to the boundaries of the plate, the problem is essentially one-dimensional. In fact, we assume tacitly that all relaxations are longitudinal in character, i.e. only interlayer spacings change when we approach the surface of the body. The simplest $1 \mathrm{D}$ atomistic model capturing these effects is a set of crystallographic planes joined by harmonic NN and NNN springs with incompatible reference lengths and distinct stiffnesses. Actually, the linear elastic constants of NN and NNN springs can even have opposite signs which may be the case when the primary interatomic potential is non-convex. The introduction of the NNN interaction in the one-dimensional theory may also be viewed as an attempt to mimic long range effects of the simpler NN models in two and three dimensions (see discussion in [P91]).

Within the framework of a one-dimensional model with compatible reference states, discrete chains with NNN interactions have already been investigated in the literature, originally, in the context of validating continuum theories with couple-stresses. Thus, Toupin and Gazis [TG64] were probably the first to study surface puckering in finite crystals with NNN interactions produced by a self-equilibrated system of surface couples. In order to describe more adequately large relative displacements of the particles near the free boundary, Gazis and Willis [GW65] considered a semi-infinite NNN chain with anharmonic forces localized in a few atomic layers near the free surfaces. Mindlin [M65] extended the above approach to the case of third nearest neighbors although in much less detail. Conditions of linear stability for an infinite crystal with NNN interaction were obtained in [GW62, GW65, K82]. More recently, [HLS92] re-derived some of these results and extended the analysis of stability to the infinite chains with third nearest neighbor interactions. Without a reference to the boundary layers, an infinite chain with non-linear NN and NNN interactions (and zero pre-stress) was studied in [JT82, J91] as a prototypical model of stable incommensurate crystal phases; two dimensional models of this type were considered in [CMV96]. Some numerical results for a finite nonlinear 
NNN chain were obtained in [TB93] where the emphasis was on fracture and phase transitions while surface relaxation was artificially suppressed.

A mismatch between the reference lengths of the NN and NNN springs has been recently introduced in Lee et al. [LSD99]. Their analysis is similar in focus to ours, albeit the fact that instead of dealing directly with the discrete model, the authors studied a long wave quasi-continuum approximation with higher strain gradients. The main price of the simplicity associated with such an approximation is that important effects carried by the discreteness of the original problem are left out. Thus, one can show that the approximate formula for the surface energy obtained in [LSD99] is valid only in the narrow range of spring stiffnesses and outside this range may differ considerably from the exact result obtained in the present paper. Another drawback of the quasi-continuum approach is that such a description is inherently non-unique. For example, a quite different quasicontinuum model with higher gradients and surface boundary layers was studied earlier by Mindlin [M65] who derived it from a discrete theory involving interaction of third neighbors. Mindlin's model was employed by Wu [W92] who computed an apparent Young's modulus for an ultra-thin plate and showed that it may be much higher than the one obtained for a continuum 3D body of the same geometry. We notice that the surface boundary layers have also been studied in the context of purely phenomenological theories with higher gradients (e.g. [C61, T63, VS99]) as well as in the framework of strongly non-local continuum models with integral spatial "memory" (e.g. [E92, K82, FM96]).

In the present paper, by assuming that the effective springs describing $\mathrm{NN}$ and NNN interactions have arbitrary reference lengths and arbitrary bulk moduli, we study a general discrete problem for a finite chain loaded by generic forces. Depending on the magnitude of the ratio of the elastic moduli, we obtain three types of solutions to the "bulk" equations: homogenous with monotone exponential boundary layers at the free surfaces, homogenous with oscillations superimposed on exponential boundary layers, and inhomogeneous (periodic), which describe commensurate and incommensurate nonBravais lattices. The solutions are simple enough and as an example we present the detailed calculations for a finite body in a soft device. Using our equilibrium solutions, we explicitly compute the surface energy and identify a contribution due to hyper-prestress. In the case when the exponential envelopes of the boundary layers overlap and the surface cannot be naturally separated from the bulk, we observe a strong dependence of the effective elastic stiffness on the external dimension of the object. In the concluding part of the paper, we argue that the consequences of the localized relaxation may also be significant outside the thin film theory; for instance, in fracture mechanics where the radius of curvature of the free surface at the tip of the crack is typically of the order of the thickness of our boundary layers. Other relevant physical phenomena include phase transitions and twinning, where the long-range interactions contribute to the formation of the boundary layers near the internal surfaces. The exponential interaction of these boundary layers plays an important role in the selection of the scale of the microstructure.

The paper is organized as follows. In Section 2, we formulate our discrete model and introduce the notion of hyper-pre-stress. In Section 3, we classify equilibrium solutions for the infinite chains and distinguish homogeneous configurations with boundary layers type growth from the periodic regimes describing multi-lattices. In Section 4, we explicitly solve the "boundary equations" for a finite chain in a 
(generalized) soft device. In Sections 5-8, we describe the inhomogeneous reference configuration, compute the effective surface energy, and study its dependence on the hyper-pre-stress and the ratio of the NN and NNN elastic moduli. In Sections 9-10, we study the size dependence of the surface energy and effective elastic moduli for the ultrathin objects. In the final Section, we summarize our findings and mention some open questions. The more technical stability analysis is left for the Appendix.

\section{The model}

Consider a one-dimensional lattice with $N+1$ identical material particles connected by $N$ elastic springs. We label the $x$-coordinate of the $k^{\text {th }}$ particle by $x_{k}$, where $0 \leq k \leq N$. We then denote by $x_{k}^{o}$, the value of the coordinate $x_{k}$, at rest (in the unloaded chain), and introduce elastic displacements from the reference configuration

$$
u_{k}=x_{k}-x_{k}^{o}
$$

We notice that the reference (rest) configuration $x_{k}^{o}$ may not be homogeneous.

Suppose that each particle in the interior of the chain interacts symmetrically with four other particles. Two of them are its nearest neighbors and the other two are its next to nearest neighbors (see Fig. 1).
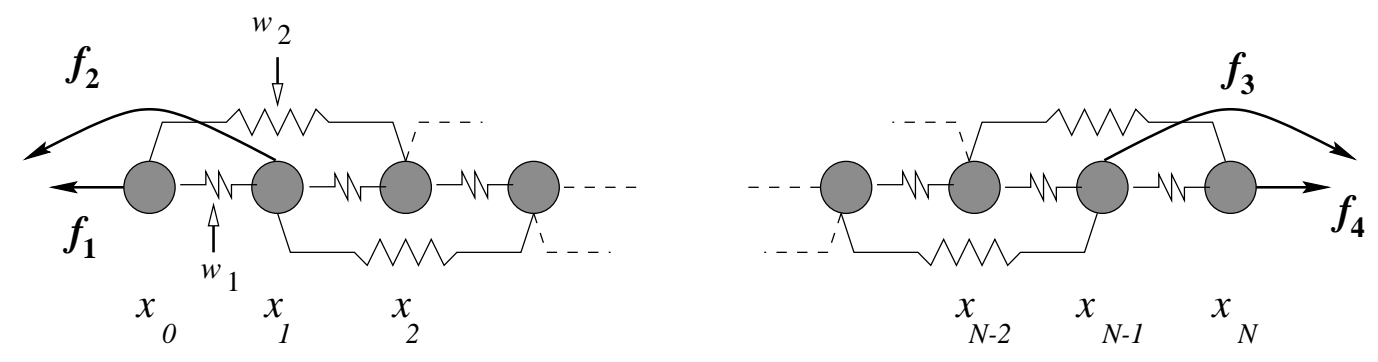

Fig.1 One-dimensional chain with next to nearest neighbor (NNN) interactions in a soft device represented by the four forces: $f_{1}, f_{2}, f_{3}, f_{4}$.

The elastic energy of the chain is defined as a sum of two terms

$$
W\left(x_{o}, \ldots, x_{N}\right)=\sum_{k=1}^{N} w_{1}\left(x_{k}-x_{k-1}\right)+\sum_{k=1}^{N-1} w_{2}\left(x_{k+1}-x_{k-1}\right)
$$

In order to be able to obtain analytical results we suppose that both functions $w_{1}$ and $w_{2}$ are quadratic. Specifically, we introduce 


$$
w_{1}(z)=\frac{\alpha}{2}\left(z-\xi_{1}\right)^{2}+w_{1}^{o}
$$

as the energy of the NN interaction and

$$
w_{2}(z)=\frac{\gamma}{2}\left(z-2 \xi_{2}\right)^{2}+w_{2}^{o}
$$

as the energy of the NNN interaction. In the formulas (2.2), (2.3) the constants $\alpha, \gamma$ are elastic stiffnesses of the springs; $\xi_{1}, 2 \xi_{2}$ are the natural spring lengths, and $w_{1}{ }^{0}, w_{2}{ }^{0}$ are the corresponding reference energies. In the generic case when $\xi_{1} \neq \xi_{2}$ the two interactions compete and the springs are pre-stressed even in the absence of the applied loads.

The energy (2.1) can be viewed as a harmonic approximation for a Lennard-Jones chain; the constants $\alpha, \gamma, \xi_{1}, \xi_{2}, w_{1}{ }^{0}$, and $w_{2}{ }^{0}$ can then be considered as adjustable parameters (see Section 11 for an explicit identification). In what follows, we will present the most general case and assume that the elastic moduli $\alpha$ and $\gamma$ may take arbitrary values, including the situations when they may be of different signs.

Contrary to the case of an NN chain, where only two boundary conditions are necessary, the NNN model requires four boundary conditions. In fact, the two missing bonds on a free surface on each side have to be replaced by either given forces or by the prescribed displacements. The following three loading devices will be relevant for our analysis:

1. Soft device. The external work takes the form

$$
Q\left(x_{o}, x_{1}, x_{N-1}, x_{N}\right)=f_{4} u_{N}+f_{3} u_{N-1}-f_{1} u_{o}-f_{2} u_{1}
$$

where we assume that $f_{p}, p=1, \ldots, 4$ are given constants satisfying the overall equilibrium condition

$$
f_{4}+f_{3}=f_{1}+f_{2}:=f
$$

2. Hard device. The particle positions $x_{o}, x_{1}, x_{N-1}$, and $x_{N}$ are prescribed.

3. Mixed device. Only the positions of the boundary particles $x_{0}$ and $x_{N}$ are prescribed. The "non-local" interaction with the loading device is modeled by the given forces $f_{2}$ and $f_{3}$ applied to the $2^{\text {nd }}$ and the $N-1^{\text {st }}$ atoms, producing the work term

$$
Q\left(x_{1}, x_{N-1}\right)=f_{3} u_{N-1}-f_{2} u_{1} \text {. }
$$

After the work of the loading device is specified, the total energy can be written as 


$$
P=W-Q,
$$

and the equilibrium equations can be obtained from the conditions $\partial P / \partial x_{k}=0$. In the case of a soft device this yields the system of $N+1$ equations which can be divided into two parts. First, we obtain the "bulk" equations

$$
0=\alpha\left(x_{k+1}+x_{k-1}-2 x_{k}\right)+\gamma\left(x_{k+2}+x_{k-2}-2 x_{k}\right)
$$

where $2 \leq k \leq N-2$. These equations must hold for the $N-3$ inner atoms that interact with their two NN and two NNN neighbors.

The four boundary atoms will also interact through forces with the loading device, providing four "boundary equations"

$$
\begin{aligned}
& 0=\alpha\left(x_{1}-x_{o}\right)+\gamma\left(x_{2}-x_{o}\right)-\left(f_{1}+\alpha \xi_{1}+2 \gamma \xi_{2}\right) \\
& 0=\alpha\left(x_{2}+x_{o}-2 x_{1}\right)+\gamma\left(x_{3}-x_{1}\right)-\left(f_{2}+2 \gamma \xi_{2}\right) \\
& 0=\alpha\left(x_{N}+x_{N-2}-2 x_{N-1}\right)+\gamma\left(x_{N-3}-x_{N-1}\right)+\left(f_{3}+2 \gamma \xi_{2}\right) \\
& 0=\alpha\left(x_{N-1}-x_{N}\right)+\gamma\left(x_{N-2}-x_{N}\right)+\left(f_{4}+\alpha \xi_{1}+2 \gamma \xi_{2}\right)
\end{aligned}
$$

Equations (2.8-12) show that the global equilibrium condition (2.5) is necessary and that the pre-stress can be interpreted as a self-equilibrated system of applied "forces". The appearance of boundary layers in the case $\xi_{1} \neq \xi_{2}$ can be attributed to the fact that these internal "forces" are compatible with the homogeneous distribution of spring lengths only if $\xi_{1}=\xi_{2}$. Finally we remark that the system of equations (2.8) can be formally "integrated" to give

$$
\alpha\left(x_{k+1}-x_{k}-\xi_{1}\right)+\gamma\left(x_{k+2}-x_{k}-2 \xi_{2}\right)+\gamma\left(x_{k+1}-x_{k-1}-2 \xi_{2}\right)=f,
$$

which is a statement that the total force (2.5) is constant throughout the length of the chain.

For the case of a hard device, equations (2. 8) will still hold, while instead of (2. 9-12) one has to prescribe the boundary displacements $u_{0}, u_{l}, u_{N-l}$, and $u_{N}$. In the case of a mixed device, two displacements must be prescribed and two "boundary" equations are to be solved.

\section{Solution of the "bulk" equations}

Since our linear system (2.8) has constant coefficients, we can use standard methods (e.g. [LL59]) to obtain an explicit solution in the form 


$$
x_{k}=\Psi_{1}(k) \quad \rho_{1}^{k}+\Psi_{2}(k) \quad \rho_{2}^{k}+\Psi_{3}(k) \quad \rho_{3}^{k}+\Psi_{4}(k) \quad \rho_{4}^{k} \text {. }
$$

Here $\rho_{q}, q=1, ., 4$, are complex roots of the characteristic equation

$$
\gamma \rho^{4}+\alpha \rho^{3}-2(\alpha+\gamma) \rho^{2}+\alpha \rho+\gamma=0 .
$$

If the root $\rho_{q}$ has multiplicity $n_{q} \geq 1$, with $\sum n_{q}=4$, then $\Psi_{q}(k)$ is the associated complex polynomial of the order $n_{q}-1$.

The characteristic equation (3.2) has a special structure and one double root is straightforward

$$
\rho_{1,2}=1
$$

The associated position field is linear

$$
x_{k}=A+B s_{k}
$$

with $A$ and $B$ arbitrary constants. Here we introduced a new (centered) labeling of the springs

$$
s_{k}=k-\frac{N}{2} .
$$

so that $A$ in (3.4) may be associated with the rigid displacement of the center of the chain. The latter may or may not coincide with the location of a particle.

The displacement field (3.4) describes a uniform distribution of particles in accordance with the Cauchy-Born hypothesis and provides a general solution for the NN chain $(\gamma=0)$. With NNN interaction added, the characteristic equation (3.2) has two other roots, which can also be found in an explicit form. First, introduce the ratio of the elastic moduli describing NN and NNN interactions

$$
\mu=\frac{\alpha}{4 \gamma}
$$

Then

$$
\rho_{3,4}=-(2 \mu+1) \pm 2 \sqrt{\mu(\mu+1)}
$$

for $\mu \leq-1$ and $\mu \geq 0$, and

$$
\rho_{3,4}=-(2 \mu+1) \pm 2 i \sqrt{-\mu(\mu+1)}
$$

for $-1 \leq \mu \leq 0$. Notice that in both cases, $\rho_{3}=\rho_{4}^{-1}$. The location of the roots $\rho_{3}$ and $\rho_{4}$ on the complex plane and their dependence on $\mu$ is illustrated in Fig.2 . 


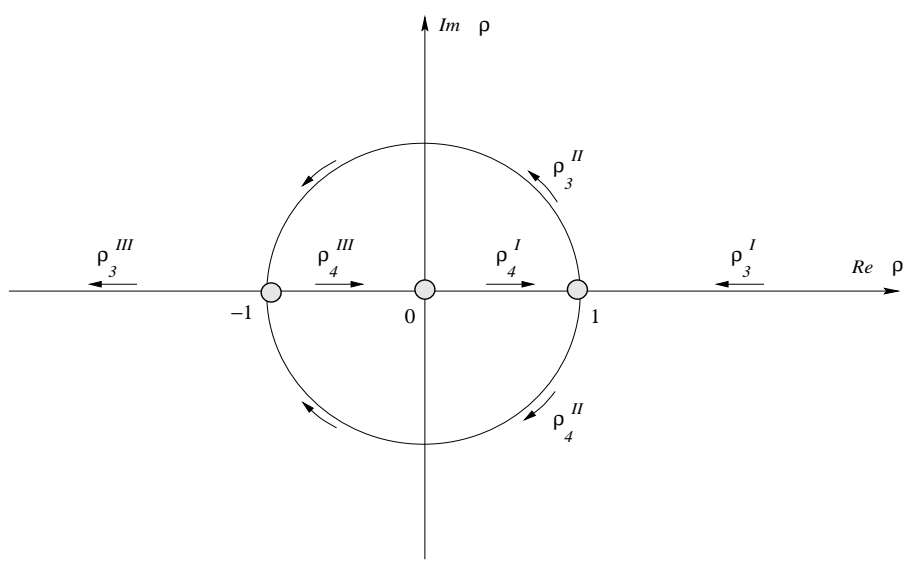

Fig. 2 - The location of the nontrivial complex roots $\rho_{3}$ and $\rho_{4}$ of the characteristic equation (3.2) at different values of the parameter $\mu$. The arrows indicate variation of the roots as $\mu$ increases from $-\infty$ to $+\infty$.

As it follows from Fig. 2 we have specified parameter $\rho_{3}$ by the additional conditions $\operatorname{Im} \rho_{3} \geq 0,\left|\rho_{3}\right| \geq 1$. It will also be convenient to introduce two other real parameters, $\lambda_{1} \geq 0$ and $\lambda_{2} \in[0, \pi]$, by the formula

$$
\rho_{3}=\exp \left(\lambda_{1}+i \lambda_{2}\right)
$$

Notice that $\lambda_{1}$, describes exponential variations of the atomic position field while $\lambda_{2}$ is responsible for the bounded periodic modulations.

In the representation of the general solution of the bulk equations different possibilities arise depending on the value of $\mu$, including three generic cases and two limiting cases, $\mu=0$ and $\mu=-1$, corresponding to the situations with a double root $\rho_{3,4}=-1$ and a fourfold root $\rho_{1,2,3,4}=1$, accordingly. Below, we give a complete list of solutions. Let $C$ and $D$ be two arbitrary real constants. Then, for the generic cases we obtain:

- $\quad-\infty<\mu<-1($ Case I)

$$
x_{k}=A+B s_{k}+C \cosh \left(\lambda_{1} s_{k}\right)+D \sinh \left(\lambda_{1} s_{k}\right)
$$

where

$$
\lambda_{1}=\ln (-(2 \mu+1)+2 \sqrt{\mu(\mu+1)}), \lambda_{2}=0
$$

(To establish relation between (3.7) and (3.1) we notice that (3.7) can also be written as

$$
x_{k}=A+B\left(k-\frac{N}{2}\right)+\left(\frac{C+D}{2}\right) \sqrt{\rho_{4}^{N}} \rho_{3}^{k}+\left(\frac{C-D}{2}\right) \sqrt{\rho_{3}^{N}} \rho_{4}^{k},
$$

and use the identities $\cosh \lambda_{1}=-(2 \mu+1)$ and $\left.\sinh \lambda_{1}=2 \sqrt{\mu(\mu+1)}\right)$; 
- $\quad-1<\mu<0$ (Case II)

$$
x_{k}=A+B s_{k}+C \cos \left(\lambda_{2} s_{k}\right)+D \sin \left(\lambda_{2} s_{k}\right)
$$

where

$$
\lambda_{1}=0, \lambda_{2}=\arg (-(2 \mu+1)+2 i \sqrt{-\mu(\mu+1)}) \in(0, \pi),
$$

which can also be written as $\cos \lambda_{2}=-(2 \mu+1)$ and $\sin \lambda_{2}=2 \sqrt{-\mu(\mu+1)}$;

- $0<\mu<+\infty$ (Case III)

$$
x_{k}=A+B s_{k}+(-1)^{k}\left[C \cosh \left(\lambda_{1} s_{k}\right)+D \sinh \left(\lambda_{1} s_{k}\right)\right]
$$

where

$$
\lambda_{1}=\ln (2 \mu+1+2 \sqrt{\mu(\mu+1)}), \lambda_{2}=\pi
$$

which can also be written as $\cosh \lambda_{1}=2 \mu+1$ and $\sinh \lambda_{1}=2 \sqrt{\mu(\mu+1)}$.

In the non-generic cases we obtain

- $\quad \mu=-1$

$$
x_{k}=A+B s_{k}+C s_{k}^{2}+D s_{k}^{3}
$$

where $\lambda_{1}=\lambda_{2}=0$,

- $\mu=0$

$$
x_{k}=A+B s_{k}+(-1)^{k}\left[C+D s_{k}\right]
$$

where $\lambda_{1}=0, \lambda_{2}=\pi$.

Each of the above position fields is a sum of a uniform particle distribution and an inhomogeneous component. The character of the inhomogeneous part of the solutions in Cases I and III is different from the one in Case II.

As it follows from (3.7), (3.11), in the generic Cases I $(\mu<-1)$ and III $(\mu>0)$ the inhomogeneous component of $x_{k}$ is exponential, which suggests formation of the boundary layers in a finite chain: the exponential "tails" will be localized near the boundaries (surface relaxations) with the "interior" particles distributed almost homogeneously. The number of springs in the boundary layers is of the order $n=1 / \lambda_{1}$; for the notion of a boundary layer to be adequate, we need $N \gg n$. As we show in Fig. 3, $n$ tends to zero as $\mu \rightarrow \pm \infty$ (NN model) and tends to infinity in the special cases when $\mu \rightarrow-1$ and $\mu \rightarrow 0$, meaning that the boundary layers spread throughout the chain. 


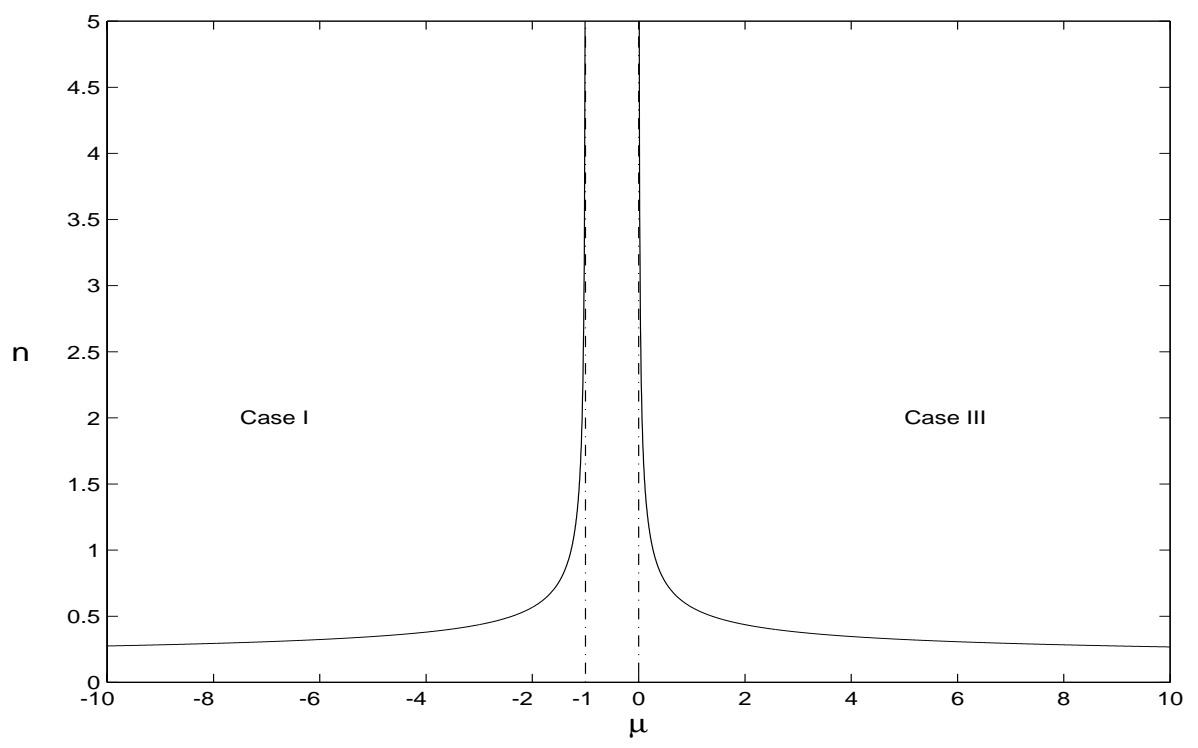

Fig.3. The number of springs $n$ inside the boundary layers as a function of $\mu$ in the Cases I and III.

In Case III the boundary layers will contain oscillations at the scale of the lattice modulated by an exponential envelope. By rearranging the terms in (3.11) we can rewrite the corresponding solution as

$$
\begin{aligned}
x_{k} & =\left[\frac{1+(-1)^{k}}{2}\right]\left[A+B s_{k}+C \cosh \left(\lambda_{1} s_{k}\right)+D \sinh \left(\lambda_{1} s_{k}\right)\right] \\
& +\left[\frac{1-(-1)^{k}}{2}\right]\left[A+B s_{k}-C \cosh \left(\lambda_{1} s_{k}\right)-D \sinh \left(\lambda_{1} s_{k}\right)\right]
\end{aligned}
$$

which reveals a superposition of two structures each analogous to (3.7).

In the generic Case II $(-1<\mu<0)$, the solution behaves quite differently and instead of exponential boundary layers, we obtain periodic configurations which may be described as a succession of stretched and compressed zones (see Fig. 4).

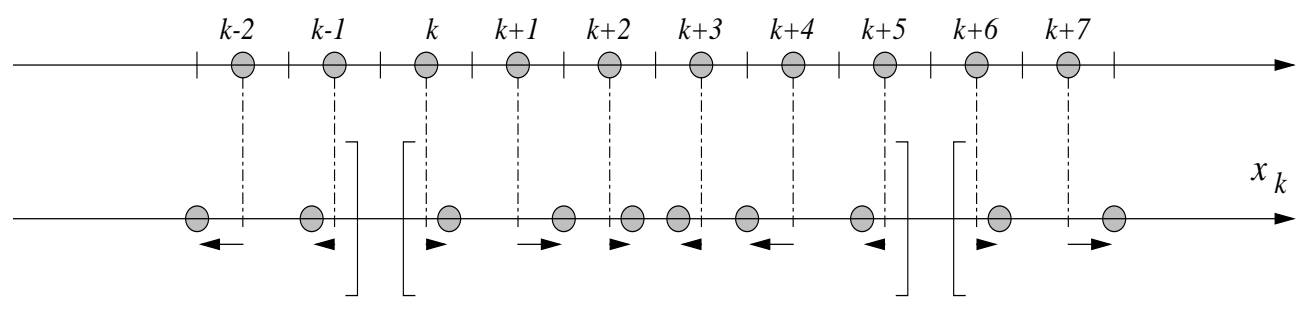

Fig. 4 A typical modulated equilibrium configuration in the Case II $(-1<\mu<0)$ representing a non-simple lattice. 
Spatial modulations of the particle density in Case II result from the presence of competing interactions ${ }^{1}$; the "frustrated" system produces non-simple (or non-Bravais) lattice which may be either commensurate or incommensurate with the periodic reference state. If the period of the inhomogeneous component of the displacement field $n=2 \pi / \lambda_{2}=p / q$ is a rational number, which takes place at $\mu=-\frac{1}{2}[1+\cos (2 \pi q / p)]$, the minimal unit cell is formed of $q$ particles. If the period is irrational, the long-range periodic order is incommensurate with an imaginary periodic lattice.

The $\mu$-dependence of the number of springs comprising a period of modulations is shown in Fig. 5. As we see, around $\mu=-1$ only very long chains can exhibit sublattices. On the contrary, around $\mu=0$ the period approaches 2 atomic distances. In the former case the sub-cell is infinite, while in the latter case it is formed exactly of 2 atoms. The last observation is in agreement with the behavior of the transitional non-generic solution. Thus, at $\mu=0$, in spite of the degeneration of the exponential "tail", the decomposition analogous to (3.15) is available

$$
x_{k}=\left(\frac{1+(-1)^{k}}{2}\right)\left[A+C+(B+D) s_{k}\right]+\left(\frac{1-(-1)^{k}}{2}\right)\left[A-C+(B-D) s_{k}\right]
$$

The position field (3.16) can be viewed as a superposition of two displaced homogeneous lattices, forming an elementary non- Bravais lattice (2-lattice).

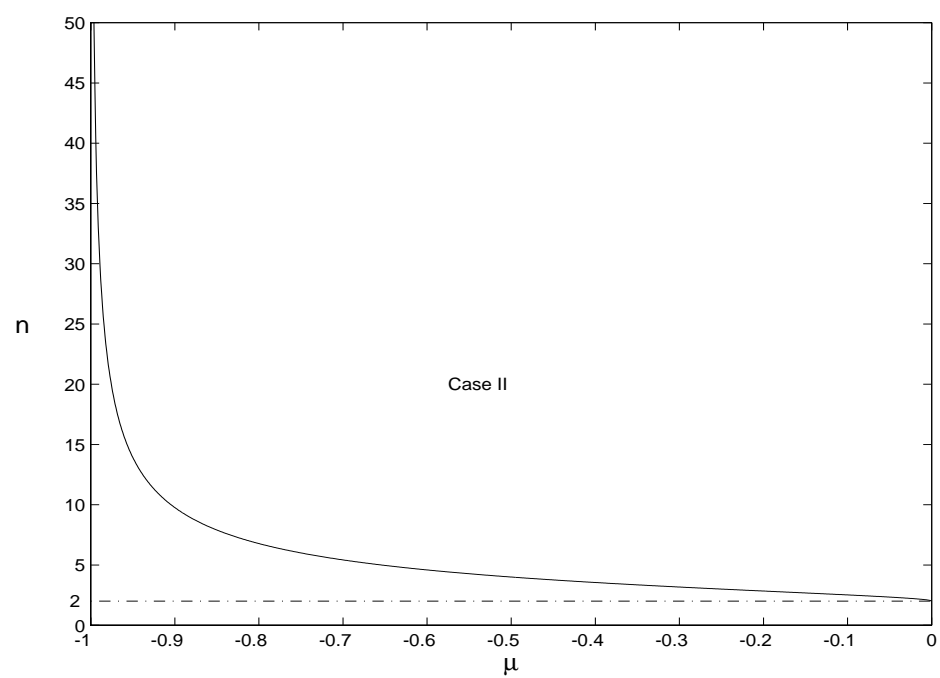

Fig. 5. A number of springs inside the period $n=2 \pi / \lambda_{2}$ as a function of $\mu$ in Case II $(-1<\mu<0)$.

\footnotetext{
${ }^{1}$ In continuum mechanical framework systems with long range competing (antiferromagnetic) interactions have been previously considered in the context of gradient models with alternating signs of higher derivatives [MPT98] and in the fully non-local integral models with sign-indeterminate kernels [RT01]. In both cases modulated structures have been obtained.
} 


\section{Finite lattice}

As we have seen in the previous section, the general solution of the "bulk" equations (2.8) is defined up to four arbitrary real constants $A, B, C$ and $D$. To specify these constants, one should use four "boundary equations" (2.9-12), which play a role of the boundary conditions. Below we show how the "boundary equations" can be applied in the case of a generic soft device.

First notice that since the four applied forces satisfy the constraint of a global equilibrium (2.5) the number of loading force parameters can be reduced to three and simultaneously one can always eliminate the overall rigid displacement of the chain by choosing $A=0$. A "natural" reduction of an arbitrary force system $\left(f_{1}, f_{2}, f_{3}, f_{4}\right)$, to the triple of independent components must respect the constraint of the overall equilibrium and be able to distinguish between the self-equilibrated contributions and the overall applied force. The application of the above constraints leads to the following result

$f_{1}=(2 \mu+1) F-F^{\prime}-F^{\prime \prime} ; \quad f_{2}=F+F^{\prime}+F^{\prime \prime} ; f_{3}=F-F^{\prime}+F^{\prime \prime} ; \quad f_{4}=(2 \mu+1) F+F^{\prime}-F^{\prime \prime}$.

This decomposition, which generalizes a construction from [TG64], is illustrated in Fig. 6. One can see that $F^{\prime}$ and $F^{\prime \prime}$ represent symmetric and anti-symmetric components of the self-equilibrated loading device, while $F$ is proportional to the total force. As we show below, the forces $F, F^{\prime}$ and $F^{\prime \prime}$ are directly associated with the coefficients $B, C$ and $D$ in the representation of the general displacement field for the infinite chain.

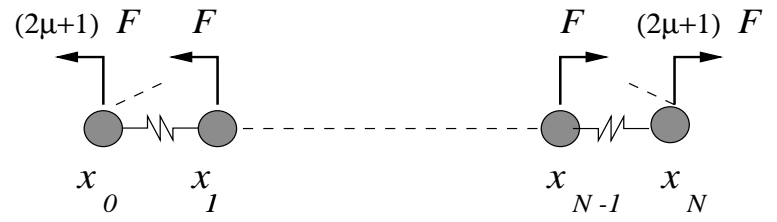

(a)

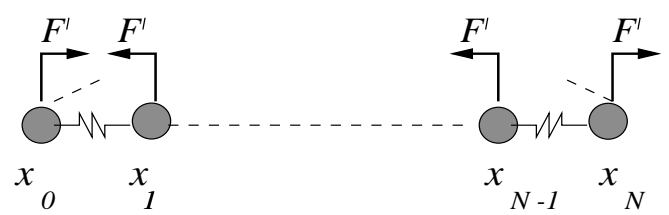

(b)

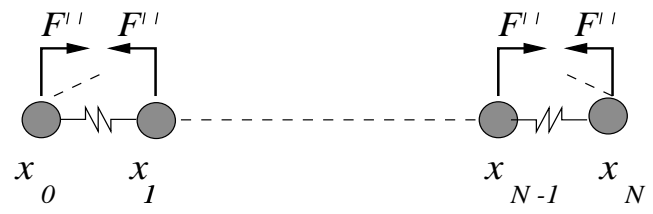

(c)

Fig. 6. Elementary force systems responsible for the homogeneous component of the strain field and for symmetric and anti-symmetric components of the boundary layers. 
We begin with the observation that the homogenous part of the position field can be presented in the form

$$
B=\frac{F}{2 \gamma}+\left(\frac{\mu-1}{\mu+1}\right) \xi+\tilde{\xi}
$$

where

$$
\xi=\frac{\xi_{1}-\xi_{2}}{2}
$$

is a characteristic of the hyper-pre-stress and

$$
\tilde{\xi}=\frac{\xi_{1}+\xi_{2}}{2}
$$

is the average of the two reference spring lengths. In the case of zero pre-stress $(\xi=0)$, parameter $\tilde{\xi}$ from (4.4) describes the lattice unit of an unloaded lattice.

From (4.2), we conclude that neither $F^{\prime}$ nor $F^{\prime \prime}$ affects the homogeneous part of the position field. Instead, these two components of a generic loading device are responsible for the formation of the boundary layers and internal modulations. Since, according to (2.9-2.12), the hyper-pre-stress is equivalent to a symmetric self-equilibrated system of applied forces, its presence will be felt outside the bulk deformation through the renormalized of $F^{\prime \prime}$ only. More specifically in each of the main cases we obtain

- $\mu<-1$

$$
\begin{aligned}
& D=\frac{\tilde{F}^{\prime \prime}}{4 \gamma \sqrt{\mu(\mu+1)} \cosh \left(\lambda_{1} N / 2\right)} \\
& C=-\frac{F^{\prime}}{4 \gamma \sqrt{\mu(\mu+1)} \sinh \left(\lambda_{1} N / 2\right)}
\end{aligned}
$$

- $\mu>0$

$$
\begin{gathered}
D=\frac{\left[\frac{(-1)^{N}-1}{2}\right] \frac{F^{\prime}}{2 \gamma}-\left[\frac{1+(-1)^{N}}{2}\right] \frac{\tilde{F}^{\prime \prime}}{2 \gamma}}{2 \sqrt{\mu(\mu+1)} \cosh \left(\lambda_{1} N / 2\right)} \\
C=\frac{\left[\frac{1+(-1)^{N}}{2}\right] \frac{F^{\prime}}{2 \gamma}+\left[\frac{1-(-1)^{N}}{2}\right] \frac{\tilde{F}^{\prime \prime}}{2 \gamma}}{2 \sqrt{\mu(\mu+1)} \sinh \left(\lambda_{1} N / 2\right)}
\end{gathered}
$$


- $-1<\mu<0$

$$
\begin{gathered}
D=\frac{\tilde{F}^{\prime \prime}}{4 \gamma \sqrt{-\mu(\mu+1)} \cos \left(\lambda_{2} N / 2\right)} \\
\left(\mu \neq-\frac{1}{2}\left(1+\cos \left(\frac{2 m-1}{N} \pi\right)\right), \quad 1 \leq m \leq \frac{N}{2}\right), \\
C=\frac{F^{\prime}}{4 \gamma \sqrt{-\mu(\mu+1)} \sin \left(\lambda_{2} N / 2\right)} \\
\left(\mu \neq-\frac{1}{2}\left(1+\cos \left(\frac{2 m}{N} \pi\right)\right), 1 \leq m<\frac{N}{2}\right) .
\end{gathered}
$$

In the above formulas we introduced a renormalized force $\tilde{F}^{\prime \prime}$ which depends on the prestress through the parameter $\xi$

$$
\tilde{F}^{\prime \prime}=F^{\prime \prime}-\left(\frac{4 \gamma \mu}{\mu+1}\right) \xi
$$

In order to rewrite the expressions (4.2), (4.5-10) in terms of the original force components, one needs to invert the force decomposition relations (4.1)

$$
\begin{gathered}
\frac{f_{3}+f_{4}}{2(\mu+1)}=F, \\
\frac{f_{4}-f_{1}}{2}=F^{\prime}, \\
\frac{(2 \mu+1) f_{3}-f_{4}}{2(\mu+1)}+\frac{f_{4}-f_{1}}{2}=F^{\prime \prime} .
\end{gathered}
$$

From these relations it is clear that $F$ is responsible for the overall force $f_{3}+f_{4}=f_{1}+f_{2}$ acting on the system. One can also notice that in the limiting case of large $|\mu|$ the NN model with $f_{1}=f_{4}$ is recovered.

The equilibrium displacement fields for all three generic Cases I, II, and III are illustrated in Fig. 7 where we present the simplest example of a body with zero external forces and non zero hyper-pre-stress. More specifically, we assume that

$$
F=0, \quad F^{\prime}=0, \quad \tilde{F}^{\prime \prime}=-\left(\frac{4 \gamma \mu}{\mu+1}\right) \xi
$$

The first two graphs exhibit monotone and oscillatory boundary layers, while the third graph demonstrates the formation of an incommensurate sub lattice. 


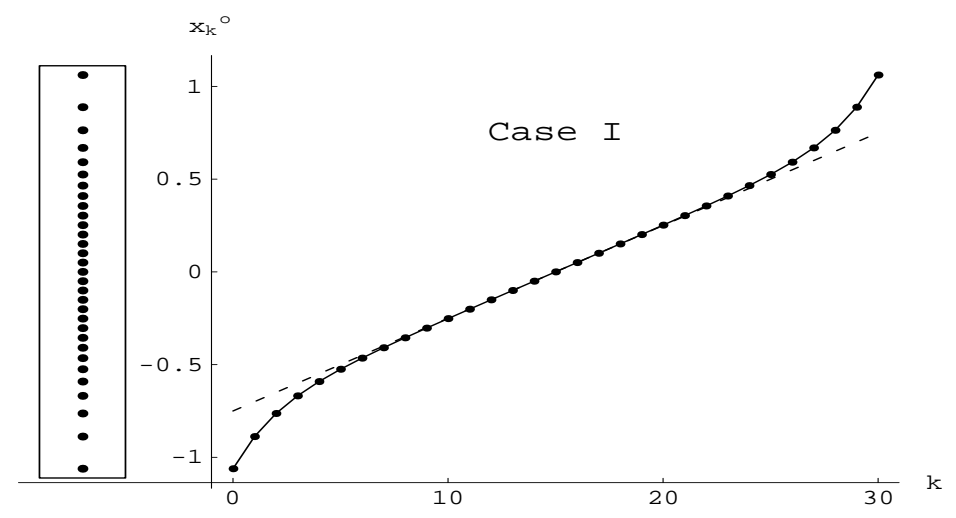

(a)

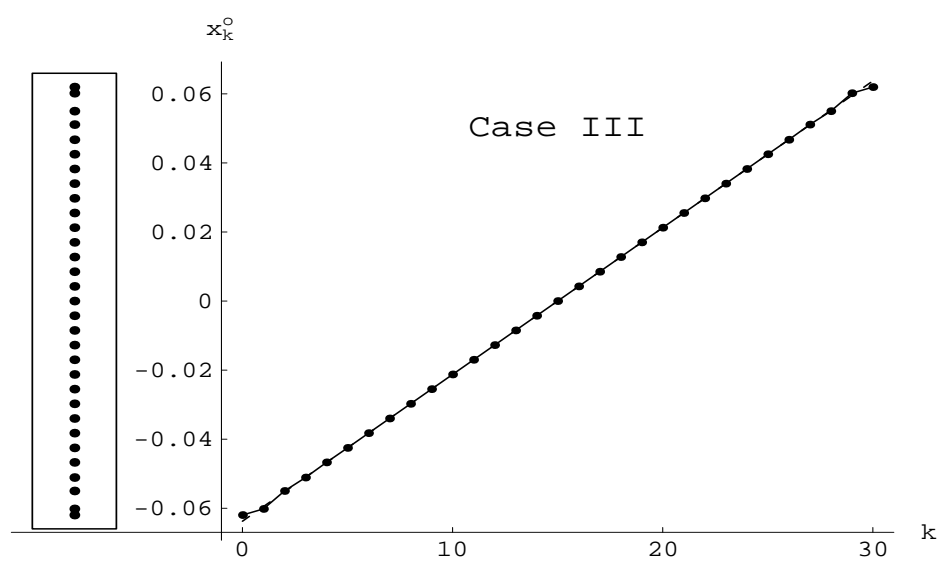

(b)

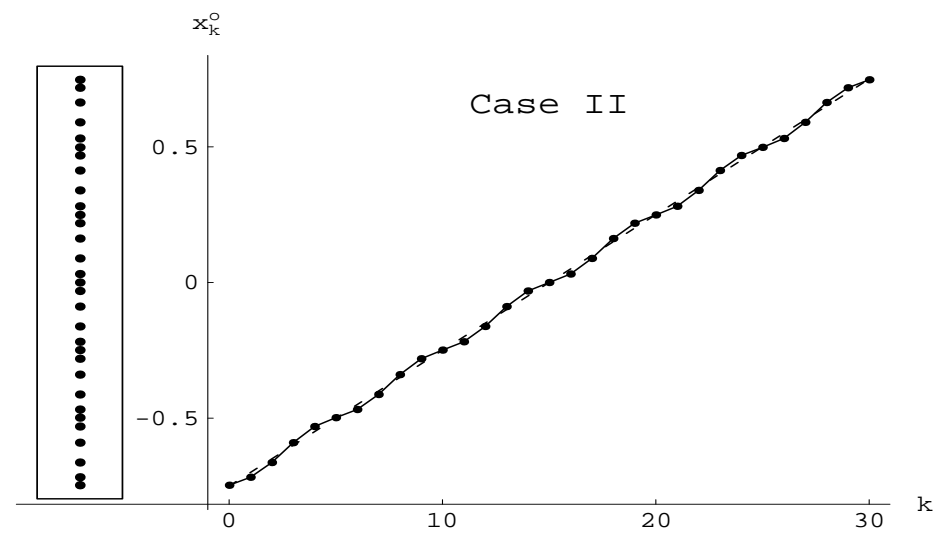

(c)

Fig. 7 Examples of the atomic positions for a hyper-pre-stressed finite chain in a reference configuration. Here $\xi=-5$ $\times .10^{-3}$ and: (a) $\mu=-1.065, \mathrm{~B}_{\mathrm{o}}=5 \times 10^{-2}$; (b) $\mu=0.1, \mathrm{~B}_{\mathrm{o}}=4 \times .10^{-3}$; (c.) $\mu=-0.65, \mathrm{~B}_{\mathrm{o}}=5 \times .10^{-2}$. 
Localized boundary layers in the Cases I and III, can be observed for sufficiently long chains when $N \lambda_{1} \gg>1$. At fixed $N$, and $\mu$ approaching 0 or -1 , the inhomogeneous boundary layers broaden and propagate inside the chain. Thus, the Taylor expansion of the solution around $\mu=-1$ takes the form

$$
\begin{aligned}
x_{k}= & {\left[\frac{f_{3}-f_{1}}{4 \gamma}+\xi_{1}+\left(\frac{f_{4}+f_{3}}{4 \gamma}-2 \xi\right)\left(\frac{2-3 N^{2}}{6}\right)\right] s_{k}+\left(\frac{f_{1}-f_{4}}{4 \gamma N}\right) s_{k}{ }^{2} } \\
& +\frac{2}{3}\left(\frac{f_{4}+f_{3}}{4 \gamma}-2 \xi\right) s_{k}{ }^{3}+O(\mu+1)
\end{aligned}
$$

Here we dropped the term of the order $|\mu+1|^{-1}$, which corresponds to the overall translation of the chain. As expected, the rest of the expansion matches exactly our solution at $\mu=-1$ (see (3.13). Similarly, around $\mu=0$ we obtain

$$
\begin{aligned}
x_{k}= & \left(\frac{f_{4}+f_{3}}{4 \gamma}+\xi_{2}\right) s_{k}+\left(\frac{(-1)^{k}-1}{2 N \mu}\right)\left[\frac{f_{4}-f_{1}}{4 \gamma}+\left(\frac{1-(-1)^{N}}{2}\right)\left(\frac{f_{3}-f_{4}}{4 \gamma}\right)\right] \\
& +(-1)^{k} s_{k}\left[\frac{f_{1}-f_{4}}{4 \gamma}+\left(\frac{1+(-1)^{N}}{2}\right)\left(\frac{f_{4}-f_{3}}{4 \gamma}\right)\right]+O(\mu)
\end{aligned}
$$

Here we preserved the singular term of the order $|\mu|^{-1}$, which describes the disintegration of our lattice into two sub-lattices. This phenomenon reflects the fact that for $\mu=0$, the NNN chain can be represented as two non-interacting NN sub-chains. As a result it is unstable unless the loading is special (meaning $f_{4}=f_{1}$ for even $N$ or $f_{3}=f_{1}$ for odd $N$ ). Up to this rigid motion, the displacement field accurately reproduces our special solution (3.14).

As we have already indicated above, in Case II $(-1<\mu \leq 0)$ the expressions for $D$ in (4.9) and $C$ in (4.10) diverge at $N$ critical (bifurcational) points

$$
\mu_{c}(q)=-\frac{1}{2}\left(1+\cos \left(\frac{q}{N} \pi\right)\right)
$$

where $q$ is an integer, $1 \leq q \leq N$. For $q$ odd, the coefficient $D$ diverges at $\mu \rightarrow \mu_{c}(q)$ when the chain is hyper- pre-stressed or if the loading is a symmetric; for $q$ even, the coefficient $C$ diverges only when the chain is loaded asymmetrically. These special values of $\mu$ mark the onset of the instability associated with the bifurcations of the periodic and quasiperiodic equilibrium configurations; the exact nature of these bifurcations depends on the nonlinear part of the model which we do not specify in the present study. The distribution of the critical points on the plane $(N, \mu)$ is illustrated in Fig. 8. Each point in 
this figure corresponds to an under-determined equilibrium configuration with the number of atoms inside a "macro-cell" equal to $2 \pi / \lambda_{2}{ }^{c}=N / q$. As $N \rightarrow \infty$, the distribution of the critical points $\mu_{c}$ becomes dense in $(-1,0]$.

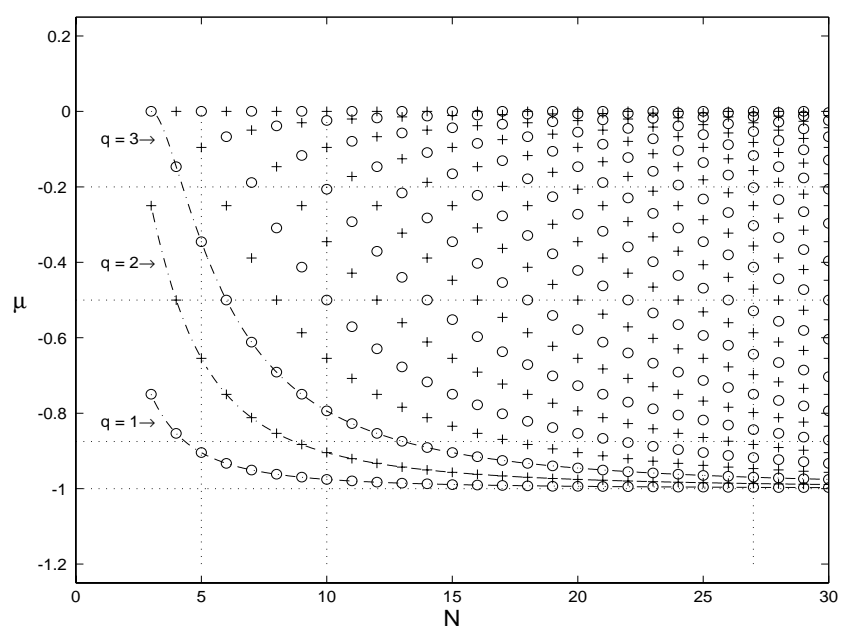

Fig. 8 - Distribution of the critical points $\mu_{c}$ (4.14) as a function of $N$, and $q$. Configurations with odd $q$ are indicated by the zeros, configurations with even $q$ - by the crosses.

Due to the fact that the bifurcation points of the equilibrium problem correspond to the points of degeneracy of the Hessian matrix (in our case the matrix of elastic stiffnesses), it is not surprising that the parameters $\mu_{c}$ play a prominent role in the analysis of stability of the equilibrium solutions. Because of the rather technical nature of this analysis, it is presented in the Appendix.

\section{Reference configuration}

As an application of the general formulas obtained in the previous section, here we consider a special case of an unloaded, self-equilibrated lattice with $f_{1}=0, f_{2}=0$, $f_{3}=0$ and $f_{4}=0$. This case is particularly interesting since it describes a reference configuration of the chain, which is in general nontrivial (see Fig. 7).

First notice that in the reference configuration

$$
B_{o}=\tilde{\xi}+\left(\frac{\mu-1}{\mu+1}\right) \xi
$$


which is the limiting value of the lattice parameter away from the free surfaces. Other coefficients $C_{0}, D_{0}$ can be readily obtained from (4.5-10). Thus, for the non oscillatory Case I $(\mu<-1)$, we obtain

$$
C_{0}=0, D_{0}=-\frac{\mu \xi}{\sqrt{\mu(1+\mu)(1+\mu) \cosh \left(\lambda_{1} N / 2\right)}}
$$

The reference strains can now be computed explicitly

$$
x_{k}^{\circ}{ }_{k}-x_{k-1}^{\circ}=B_{o}+2 \xi\left(\frac{\mu}{1+\mu}\right)\left(\frac{\cosh \left(\frac{2 s_{k}-1}{2} \lambda_{1}\right)}{\sqrt{-\mu} \cosh \left(\lambda_{1} N / 2\right)}\right) .
$$

From (5.2) one can see that at large $N$ the particle spacing approaches the constant value $B_{0}$. One can also notice that if $\xi>0(\xi<0)$ the length of the springs is necessarily shorter (longer) near the surface than inside the chain.

Although such simple conclusions can not be reached for the oscillatory Case III $(\mu>0)$, we can still estimate the deviation of the spring lengths from their homogeneous value $B_{o}$. We obtain

$$
\left|x^{\circ}{ }_{k}-x^{\circ}{ }_{k-1}-B_{o}\right|=2|\xi|\left(\frac{\mu}{1+\mu}\right)\left(\frac{\cosh \left(\frac{2 s_{k}-1}{2} \lambda_{1}\right)}{\sqrt{\mu} \sinh \left(\lambda_{1} N / 2\right)}\right),
$$

for $N$ even and

$$
\left|x^{\circ}{ }_{k}-x^{\circ}{ }_{k-1}-B_{o}\right|=2|\xi|\left(\frac{\mu}{1+\mu}\right)\left(\frac{\sinh \left(\left|\frac{2 s_{k}-1}{2}\right| \lambda_{1}\right)}{\sqrt{\mu} \cosh \left(\lambda_{1} N / 2\right)}\right),
$$

for $N$ odd. As it follows from $(5.3,5.4)$, these deviations are necessarily larger near the free surfaces than inside the lattice.

Now, for the total length of the chain in the reference configuration we obtain

$$
L_{o}=N B_{o}+2 \xi\left(\frac{\mu}{\mu+1}\right)^{3 / 2} \frac{\tanh \left(\lambda_{1} N / 2\right)}{\mu}
$$

at $\mu<-1$ and 


$$
L_{o}=N B_{o}+\frac{2 \xi}{\mu}\left(\frac{\mu}{\mu+1}\right)^{3 / 2}\left(\left(\frac{1+(-1)^{N}}{2}\right) \tanh \left(\lambda_{1} N / 2\right)+\left(\frac{1-(-1)^{N}}{2}\right) \tanh ^{-1}\left(\lambda_{1} N / 2\right)\right)
$$

at $\mu>0$. One can see, that for $\mu<-1$ and $\xi>0(\xi<0)$, the relaxed chain is shorter (longer) than the equivalent homogeneous lattice without the boundary layers. When $\mu>0$, the chain with boundary layers is longer (shorter) for $\xi>0(\xi<0)$. Now, since for $\lambda_{1} N>>1$ we can approximate

$$
\tanh \left(\lambda_{1} N / 2\right) \approx 1-2 \exp \left(-2 \lambda_{1} N / 2\right)
$$

the difference between the two lengths, mentioned above, behaves asymptotically as

$$
L_{o}-N B_{o} \approx 2 \xi\left(\frac{\mu}{\mu+1}\right)^{3 / 2}\left(\frac{1-2 \exp \left(-\lambda_{1} N\right)}{\mu}\right)
$$

for $\mu<-1$ and

$$
L_{o}-N B_{o} \approx 2 \xi\left(\frac{\mu}{\mu+1}\right)^{3 / 2}\left(\frac{1-2(-1)^{N} \exp \left(-\lambda_{1} N\right)}{\mu}\right)
$$

for $\mu>0$. As $N \rightarrow \infty$ or $\mu \rightarrow \infty$ the right hand sides of these expressions tend to a constant, which means that for sufficiently large $N$,

$$
L_{o} \approx N B_{o} \text {. }
$$

This, of course, agrees with the classical continuum theory, which characteristically assumes that the reference configuration is homogeneous.

\section{The total energy}

In this section, we calculate the total elastic energy of the equilibrium chain and specify the contribution associated with the surface energy. We use the definition

$$
W=N w_{1}^{o}+(N-1) w_{2}^{o}+\frac{\alpha}{2} \sum_{k=1}^{N}\left(x_{k}-x_{k-1}-\xi_{1}\right)^{2}+\frac{\gamma}{2} \sum_{k=1}^{N-1}\left(x_{k+1}-x_{k-1}-2 \xi_{2}\right)^{2}
$$


This expression can be rewritten as

$$
W=W_{\mathrm{o}}+W_{1}+W_{2}
$$

where

$$
W_{o}=N w_{1}^{o}+(N-1) w_{2}^{o}+\frac{\alpha}{2} \sum_{k=1}^{N}\left(x_{k}^{o}-x_{k-1}^{o}-\xi_{1}\right)^{2}+2 \gamma \sum_{k=1}^{N-1}\left(\frac{x_{k+1}^{o}-x_{k-1}^{o}}{2}-\xi_{2}\right)^{2},
$$

is the energy of the reference state;

$$
W_{1}=\alpha \sum_{k=1}^{N}\left(u_{k}-u_{k-1}\right)\left(x_{k}^{o}-x_{k-1}^{o}-\xi_{1}\right)+\gamma \sum_{k=1}^{N-1}\left(u_{k+1}-u_{k-1}\right)\left(x_{k+1}^{o}-x_{k-1}^{o}-2 \xi_{2}\right)
$$

is the linear coupling term and finally

$$
W_{2}=\frac{\alpha}{2} \sum_{k=1}^{N}\left(u_{k}-u_{k-1}\right)^{2}+2 \gamma \sum_{k=1}^{N-1}\left(\frac{u_{k+1}-u_{k-1}}{2}\right)^{2}
$$

is the quadratic energy of the elastic deformation due to the external loading. To compute the energy of a given equilibrium configuration we must substitute in (6.1) the values of the equilibrium displacements $x_{k}$. For determinacy, in what follows we consider the chain in a soft device and use our explicit solution (4.2-10). According to the Clapeyron's theorem,

$$
Q=2 W_{2}
$$

and therefore, whenever the elastic energy (6.2) is known, the calculation of the total energy is straightforward.

We first notice that due to the condition of equilibrium, our linear coupling term in the energy is equal to zero

$$
W_{1}=\sum_{k=0}^{N} u_{k} \frac{\partial P}{\partial x_{k}}\left(x_{k}^{o}\right) \equiv 0
$$


To compute two other terms -- the reference energy $W_{0}$ and the elastic energy $W_{2}-$ it is convenient to make some changes in the representation of the general solution of the equilibrium equations. For simplicity, we shall illustrate the method for the Case II $(-1<\mu$ $<0)$.

Let us rewrite the expressions for the reference particle configuration and for the displacement field due to the applied forces in the common form

$$
\begin{aligned}
& x_{k}^{\circ}=B_{o} s_{k}+X_{o} \sin \left(k \lambda_{2}+\varphi_{o}\right), \\
& u_{k}=B^{\prime} s_{k}+X^{\prime} \sin \left(k \lambda_{2}+\varphi\right) .
\end{aligned}
$$

Here $X^{\prime}, B^{\prime}$ and $\varphi$ are real constants satisfying

$$
\begin{aligned}
& B^{\prime}=B-B_{0}, \\
& C^{\prime}=C-C_{o}=X \sin \left(\frac{N}{2} \lambda_{2}+\varphi\right), \\
& D^{\prime}=D-D_{o}=X^{\prime} \cos \left(\frac{N}{2} \lambda_{2}+\varphi\right) .
\end{aligned}
$$

The constants $X_{o}$, and $\varphi_{o}$ characterizing the reference state can be obtained from

$$
\begin{gathered}
C_{o}=X_{o} \sin \left(\frac{N}{2} \lambda_{2}+\varphi_{o}\right)=0, \\
D_{o}=X_{o} \cos \left(\frac{N}{2} \lambda_{2}+\varphi_{o}\right)=-\left(\frac{2 \mu}{\mu+1}\right) \frac{\xi}{\cos \left(\frac{N}{2} \lambda_{2}\right) \sin \lambda_{2}} .
\end{gathered}
$$

In order to obtain analogous representation for the equilibrium solutions in the other two Cases I and III, we do not need to do additional computations. In Case I, it is sufficient to replace in the final formulas

$$
\begin{gathered}
\lambda_{2} \rightarrow i \lambda_{1}, \\
\varphi \rightarrow i \varphi, \\
C \rightarrow C, \\
D \rightarrow-i D .
\end{gathered}
$$

In Case III, the replacement formulas are as follows

$$
\begin{gathered}
\lambda_{2} \rightarrow \pi+i \lambda_{1}, \\
\varphi \rightarrow i \varphi, \\
C \rightarrow C \cos (\pi N / 2)-i D \sin (\pi N / 2), \\
D \rightarrow-C \sin (\pi N / 2)-i D \cos (\pi N / 2) .
\end{gathered}
$$


For simplicity in we provide the detailed calculations in Case II only. For the other two main cases, the final expressions can be easily obtained by the above substitutions.

\section{The reference energy}

In order to calculate the reference elastic energy $W_{o}$ in the Case II $(-1<\mu<0)$, we substitute (6.7) into (6.3) to obtain

$$
\begin{gathered}
\frac{W_{o}}{2 \gamma}=\frac{N w_{1}^{o}+(N-1) w_{1}^{o}}{2 \gamma}+N \mu\left(B_{o}-\xi_{1}\right)^{2}+(N-1)\left(B_{o}-\xi_{2}\right)^{2} \\
\left.\left.+X_{o}\left[-\left(\frac{B_{o}-\xi_{1}}{2}\right) \sum_{k=1}^{N}\left(\sin (k+1) \lambda_{2}+\varphi_{o}\right)-\sin (k-1) \lambda_{2}+\varphi_{o}\right)+\sin \left(k \lambda_{2}+\varphi_{o}\right)-\sin (k-2) \lambda_{2}+\varphi_{o}\right)\right) \\
\left.\left.+\left(B_{o}-\xi_{2}\right) \sum_{k=1}^{N-1}\left(\sin \left((k+1) \lambda_{2}+\varphi_{o}\right)-\sin (k-1) \lambda_{2}+\varphi_{o}\right)\right)\right] \\
+\frac{X_{o}^{2}}{2} \sin \lambda_{2}\left[-\sin \lambda_{2}-\sum_{k=1}^{N}\left(\frac{\sin 2\left(k \lambda_{2}+\varphi_{o}\right)-\sin 2\left((k-1) \lambda_{2}+\varphi_{o}\right)}{2}\right)+\sum_{k=1}^{N-1}\left(\frac{\sin \left((2 k+1) \lambda_{2}+2 \varphi_{o}\right)-\sin \left((2 k-1) \lambda_{2}+2 \varphi_{o}\right)}{2}\right)\right]
\end{gathered}
$$

Due to the mutual cancellations, the trigonometric sums in (7.1) can be computed explicitly

$$
\begin{aligned}
\frac{W_{o}}{2 \gamma}= & \frac{N w_{1}^{o}+(N-1) w_{2}^{o}}{2 \gamma}+N \mu\left(B_{o}-\xi_{1}\right)^{2}+(N-1)\left(B_{o}-\xi_{2}\right)^{2} \\
& +\left(1+\cos \lambda_{2}\right)\left(\xi_{1}-\xi_{2}\right) X_{o}\left[\sin \left(N \lambda_{2}+\varphi_{o}\right)-\sin \varphi_{o}\right]+\left(B_{o}-\xi_{2}\right)^{2} \\
& -\frac{1}{2}\left[B_{o}-\xi_{2}+X_{o} \sin \left(N \lambda_{2}+\varphi_{o}\right) \sin \lambda_{2}\right]^{2}-\frac{1}{2}\left[B_{o}-\xi_{2}+X_{o} \sin \varphi_{o} \sin \lambda_{2}\right]^{2} \\
& -\frac{\left(1-\cos \lambda_{2}\right) X_{o}^{2}}{4} \sin \lambda_{2}\left[\sin 2\left(N \lambda_{2}+\varphi_{o}\right)-\sin 2 \varphi_{o}\right] .
\end{aligned}
$$

By rewriting (7.2) in terms of the original constants $C_{0}, D_{0}$, we obtain

$$
\begin{aligned}
\frac{W_{o}}{2 \gamma}= & \frac{N w_{1}^{o}+(N-1) w_{2}^{o}}{2 \gamma}+N\left[\mu\left(B_{o}-\xi_{1}\right)^{2}+\left(B_{o}-\xi_{2}\right)^{2}\right]-4 \mu\left(\xi_{1}-\xi_{2}\right) D_{o} \sin \left(\frac{N \lambda_{2}}{2}\right) \\
& -\left[B_{o}-\xi_{2}+D_{o} \cos \left(\frac{N \lambda_{2}}{2}\right) \sin \lambda_{2}\right]^{2}-(\mu+1) D_{o}^{2} \sin N \lambda_{2} \sin \lambda_{2} \\
& \left.+\frac{C_{o}^{2}}{2} \sin \lambda_{2}\left(\sin N \lambda_{2}-\sin (N+1) \lambda_{2}+\sin \lambda_{2}\right)\right) .
\end{aligned}
$$


Finally, after replacing $B_{o}, C_{o}$ and $D_{o}$ by the corresponding expressions $(5.1,6.13,6.13)$, we obtain

$$
W_{o}(N)=N w_{1}^{o}+(N-1) w_{2}^{o}+2 \alpha \xi^{2}\left(\frac{1}{\mu+1}\right)\left(N-\sqrt{\frac{-\mu}{\mu+1}} \tan \frac{N \lambda_{2}}{2}\right) .
$$

This is a desired formula for the reference energy. Notice that the first two terms characterize the ground-state cohesive energy while the last term represents the contribution due to the pre-stress.

By using the replacement rules formulated in the end of Section 6, we can obtain formulas for the reference energy in Cases I and III. Thus, for $\mu<-1$ we get

$$
W_{o}=N w_{1}^{o}+(N-1) w_{2}^{o}+2 \alpha \xi^{2}\left(\frac{1}{\mu+1}\right)\left(N-\sqrt{\frac{\mu}{\mu+1}} \tanh \frac{N \lambda_{1}}{2}\right) \text {. }
$$

For $\mu>0$ we obtain

$$
\begin{aligned}
& W_{o}(N)=N w_{1}^{o}+(N-1) w_{2}^{o} \\
& +2 \alpha \xi^{2}\left(\frac{1}{\mu+1}\right)\left[N-\sqrt{\frac{\mu}{\mu+1}}\left[\left(\frac{1+(-1)^{N}}{2}\right) \tanh \frac{N \lambda_{1}}{2}+\left(\frac{1-(-1)^{N}}{2}\right) \tanh ^{-1} \frac{N \lambda_{1}}{2}\right]\right] .
\end{aligned}
$$

At large $N$ the energy in Cases I and III approaches a linear function. On the contrary, at small $N$ the deviations from the linearity are substantial, pointing towards a characteristic size effect due to the overlapping of the boundary layers. In Case II the energy at large $N$ oscillates periodically around a linear function, exhibiting singularities near the bifurcational points $N\left(\mu_{c}\right)$ (see (4.14)).

\section{Surface and fracture energies}

In this section by subtracting from the reference energy of a finite body $W_{0}$ the "bulk" part $W_{0}{ }^{b}$, we compute the surface energy of a chain with NNN interaction. It is natural to interpret the bulk energy $W_{0}{ }^{b}$ as the energy of the uniform distribution of 
particles with the lattice parameter equal to $B_{0}$ (see (5.1)). To compute the bulk energy for a finite chain we should take the energy of the shared bonds into account. We obtain

$$
\begin{aligned}
W_{o}^{b}= & \sum_{k=1}^{N} w_{1}\left(x_{k}-x_{k-1}\right)+\sum_{k=1}^{N-1} w_{2}\left(x_{k+1}-x_{k-1}\right) \\
& +\frac{1}{2} w_{1}\left(x_{o}-x_{-1}\right)+\frac{1}{2} w_{2}\left(x_{1}-x_{-1}\right)+\frac{1}{2} w_{2}\left(x_{o}-x_{-2}\right) \\
& +\frac{1}{2} w_{1}\left(x_{N+1}-x_{N}\right)+\frac{1}{2} w_{2}\left(x_{N+1}-x_{N-1}\right)+\frac{1}{2} w_{2}\left(x_{N+2}-x_{N}\right)
\end{aligned}
$$

By substituting the definitions of $w_{1}, w_{2}$ and using our ansatz $x_{k}^{o}=B_{o} s_{k}$ for the uniform reference displacement field, we obtain

$$
W_{o}^{b}=(N+1)\left[w_{1}^{o}+w_{2}^{o}+2 \alpha\left(\frac{1}{\mu+1}\right) \xi^{2}\right]
$$

Now the surface energy associated with each of the free surfaces can be computed from the formula

$$
E_{s}=\frac{W_{o}-W_{o}^{b}}{2}
$$

By using expressions for $W_{0}$ from the previous Section, and introducing the notation

$$
E_{s}^{o}=-\frac{1}{2} w_{1}^{o}-w_{2}^{o}
$$

we obtain

$$
E_{s}=E_{s}^{o}-\alpha \xi^{2}\left(\frac{1}{1+\mu}\right)\left(1+\sqrt{\frac{\mu}{\mu+1}} \tanh \frac{N \lambda_{1}}{2}\right)
$$

at $\mu<-1$ and

$$
E_{s}=E_{s}^{o}-\alpha \xi^{2}\left(\frac{1}{1+\mu}\right)\left[1+\sqrt{\frac{\mu}{1+\mu}}\left[\left(\frac{1+(-1)^{N}}{2}\right) \tanh \frac{N \lambda_{1}}{2}+\left(\frac{1-(-1)^{N}}{2}\right) \tanh ^{-1} \frac{N \lambda_{1}}{2}\right]\right]
$$

at $\mu>0$. The typical graphs of the hyper-pre-stress related part of the surface energy $E_{s}{ }^{\xi}=E_{s}-E_{s}{ }^{o}$ versus the number of particles $N$ are presented in Fig. 9 at different values of $\mu$. For determinacy, here and in what follows we assumed that $\alpha>0$. One can see that the short chains exhibit size effect. For sufficiently long chains $E_{s}$ approaches a constant value which would commonly be associated with the surface energy. 


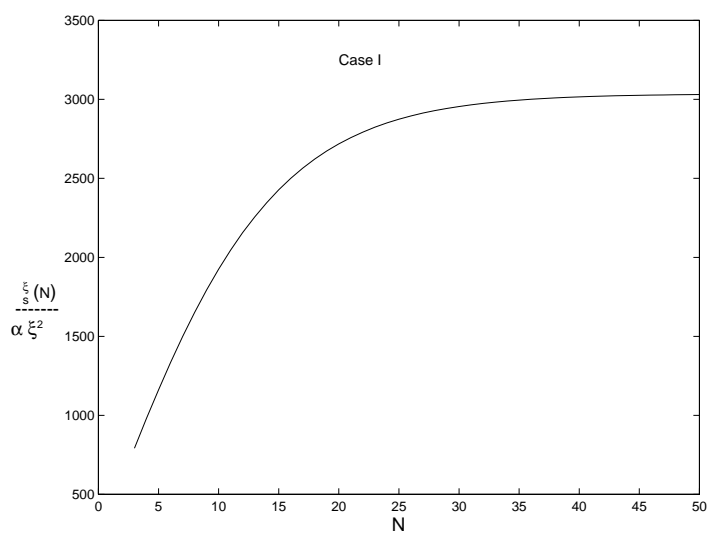

(a)

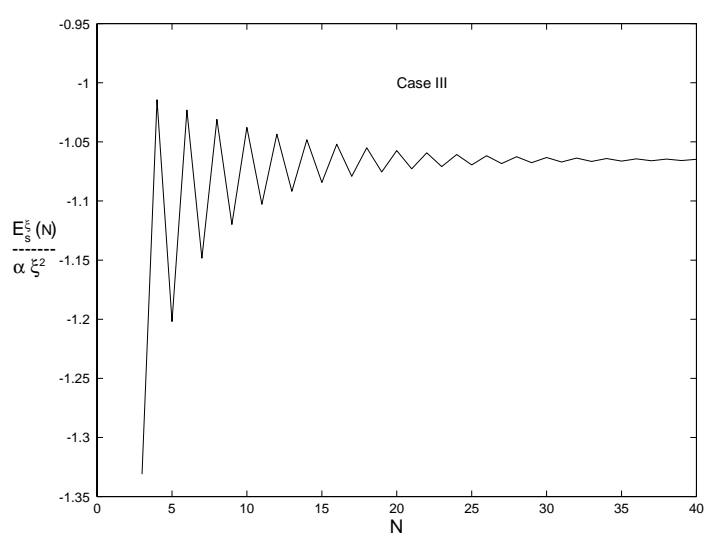

(b)

Fig. 9 The dependence of the prestress-related part of the surface energy of a finite chain on the number of particles $N$. (a) $\mu=-1.005$, (b) $\mu=0.005$.

Specifically, in the limit $N \lambda_{1} \rightarrow \infty$ for both Cases I and III we obtain

$$
\tilde{E}_{s}=-\frac{1}{2} w_{1}^{o}-w_{2}^{o}-\alpha \xi^{2}\left(\frac{1}{1+\mu}\right)\left[1+\sqrt{\frac{\mu}{1+\mu}}\right]
$$

This expression can be interpreted as (one half of ) the total energy of fracture associated with breaking the bond between the two adjacent half-spaces. This energy consists of two contributions

$$
\tilde{E}_{s}=E_{s}^{o}+\tilde{E}_{s}^{\xi}
$$


The first term $E_{s}{ }^{o}$ (see (8.4)) is the standard "pre-stress-free" surface energy. One can expect this term to be positive. The second term

$$
\tilde{E}_{s}^{\xi}=-\alpha \xi^{2}\left(\frac{1}{1+\mu}\right)\left[1+\sqrt{\frac{\mu}{1+\mu}}\right],
$$

which is entirely due to the pre-stress, is plotted in Fig. 10 as a function of $\mu$. One can see that it is positive in the non-oscillatory Case $\mathrm{I}(\mu<-1)$ and is negative in the oscillatory Case III $(\mu>0)$.

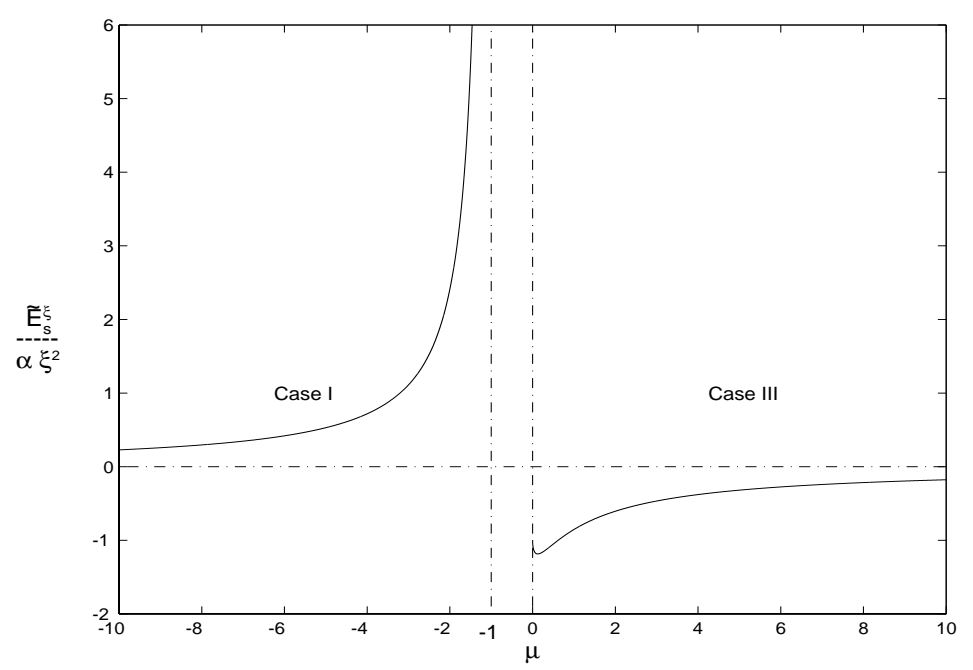

Fig.10 The $\mu$ dependence of the normalized surface energy for an infinite chain.

Let us consider the process of the creation of new free surfaces in more detail. When the material on one side of the fracture plane is removed, the internal forces on that surface are no longer balanced. The new equilibrium configuration has a region close to the surface which is strained with respect to the bulk. To avoid this relaxation, one can impose a system of forces preventing boundary layers from forming. The net force must be zero so this system of forces must be self-equilibrating. By using (5.1), it is not hard to show that it amounts to applying a couple

$$
f_{1}=-\frac{4 \gamma \mu \xi}{1+\mu} \quad, \quad f_{2}=\frac{4 \gamma \mu \xi}{1+\mu}
$$

In terms of the generalized force components (4.1), we obtain 


$$
F=0, F^{\prime}=0, F^{\prime \prime}=\frac{4 \gamma \mu \xi}{1+\mu}
$$

The energy of a "free" surface with the forces (8.10) applied is equal to

$$
\tilde{E}_{s}^{o}=E_{s}^{o}-\alpha \xi^{2} \frac{1+2 \mu}{(1+\mu)^{2}}
$$

This energy has a cohesive contribution $E_{s}{ }^{\circ}$ and another term due to the hyper-pre-stress interaction with the deformation in the bulk. Now, if we remove forces (8.10), the relaxation near the surface will follow, leading to the formation of the boundary layers. The corresponding energy change can be computed by subtracting (8.12) from (8.7). We obtain

$$
\tilde{E}_{s}-\widetilde{E}_{s}^{o}=\alpha \xi^{2} \frac{1}{1+\mu} \sqrt{\frac{\mu}{1+\mu}}\left(\sqrt{\frac{\mu}{1+\mu}}-1\right)
$$

The right hand side of this expression represents the surface energy contribution due solely to the boundary layers. Intuitively, one can expect that the presence of a surface allows the system to lower its free energy by changing its configuration in a region near the surface from its bulk configuration; as a result, free energy change associated with the surface relaxation must be negative. Analysis of the formula (8.13) shows that this is in fact what is happening in both Cases I and III.

\section{The elastic energy}

Now we consider the elastic energy $W_{2}$ which is entirely due to the external loading. The computations here are exactly analogous to the ones in Section 7 with parameters $\xi_{1}$, $\xi_{2}, w_{1}^{o}, w_{2}^{o}$ dropped, and the constants $\left(B_{o}, C_{o}, D_{o}\right)$ replaced by $\left(B^{\prime}, C^{\prime}, D^{\prime}\right)$. Starting as before with Case II $(-1<\mu<0)$ we obtain

$$
\begin{gathered}
W_{2}=2 \gamma(\mu+1)\left(N B^{\prime 2}-D^{\prime 2} \sin N \lambda_{2} \sin \lambda_{2}\right)-2 \gamma\left(B^{\prime}+D^{\prime} \cos \frac{N \lambda_{2}}{2} \sin \lambda_{2}\right)^{2} \\
+2 \gamma C^{\prime 2} \sin \frac{N \lambda_{2}}{2} \sin \lambda_{2}\left[\cos \frac{N \lambda_{2}}{2}-\cos \left(\frac{N}{2}-1\right) \lambda_{2}\right] .
\end{gathered}
$$

For the other generic cases the formulas for $W_{2}$ are analogous. Thus, in Case I $(\mu<-1)$ 


$$
\begin{gathered}
W_{2}=2 \gamma(\mu+1)\left(N B^{\prime 2}-D^{\prime 2} \sinh N \lambda_{1} \sinh \lambda_{1}\right)-2 \gamma\left(B^{\prime}+D^{\prime} \cosh \frac{N \lambda_{1}}{2} \sinh \lambda_{1}\right)^{2} \\
-2 \gamma C^{\prime 2} \sinh \frac{N \lambda_{1}}{2} \sinh \lambda_{1}\left[\cosh \frac{N \lambda_{1}}{2}-\cosh \left(\frac{N}{2}-1\right) \lambda_{1}\right]
\end{gathered}
$$

and in Case III $(\mu>0)$

$$
\begin{aligned}
W_{2}=2 \gamma(\mu+1) & {\left[N B^{\prime 2}+\left(D^{\prime 2}+C^{2}\right) \sinh N \lambda_{1} \sinh \lambda_{1}\right]-\gamma\left[B^{\prime}-(-1)^{N}\left(D^{\prime} \cosh \frac{N \lambda_{1}}{2}+C^{\prime} \sinh \frac{N \lambda_{1}}{2}\right) \sinh \lambda_{1}\right]^{2} . } \\
& -\gamma\left[B^{\prime}-\left(D^{\prime} \cosh \frac{N \lambda_{1}}{2}-C^{\prime} \sinh \frac{N \lambda_{1}}{2}\right) \sinh \lambda_{1}\right]^{2}
\end{aligned}
$$

The explicit value of the energy depends on the coefficients $B^{\prime}, C^{\prime}, D^{\prime}$, which in turn depend on the specifics of the loading device. As an example, consider a (particular) hard device providing overall strain $\bar{\varepsilon}$

$$
u_{N}=u_{N-1}=-u_{1}=-u_{o}=\frac{N}{2} B_{o} \bar{\varepsilon} .
$$

For these boundary conditions, the equilibrium problem can be solved explicitly (for details see [C00]). In Case I $(-1<\mu<0)$ we obtain

$$
\begin{gathered}
B^{\prime}=N B_{o} \bar{\varepsilon}\left(\frac{\sinh \left(\frac{N}{2}-1\right) \lambda_{1}-\sinh \frac{N \lambda_{1}}{2}}{N \sinh \left(\frac{N}{2}-1\right) \lambda_{1}-(N-2) \sinh \frac{N \lambda_{1}}{2}}\right) \\
D^{\prime}=\frac{N B_{o} \bar{\varepsilon}}{N \sinh \left(\frac{N}{2}-1\right) \lambda_{1}-(N-2) \sinh \frac{N \lambda_{1}}{2}} \\
C^{\prime}=0
\end{gathered}
$$

To calculate the energy $W_{2}$ we substitute the values of the coefficients $B^{\prime}, C^{\prime}, D^{\prime}$, from (9.5-7) into (9.2). After straightforward calculation we obtain

$$
W_{2}(\bar{\varepsilon})=N B_{0} \frac{E \bar{\varepsilon}^{2}}{2}\left(1+\frac{2 \tanh \frac{N \lambda_{1}}{2}}{2[N(\mu+1)-1] \tanh \frac{N \lambda_{1}}{2}+N \sinh \lambda_{1}}\right)
$$

Here we introduced the overall bulk modulus of an infinite chain

$$
E=4 \gamma(\mu+1) B_{o}=(\alpha+4 \gamma) B_{o}
$$


For other generic Cases II $(-1<\mu<0)$ and III $(\mu>0)$ the expressions for the energy are similar. The dependence of the energy $W_{2}$ from (9.8) on the number of particles is illustrated in Fig. 11. One can see that at large $N$, the energy is proportional to the number of particles as in a conventional continuum theory; this assumption leads to a classical scaling for the energy of a thin plate. However, at sufficiently small $N$, there is a pronounced size effect characterized by a nonlinear and non-monotone dependence of the energy on $N$. This observation suggests that for ultra-thin plates with the thickness of the order of internal length scale (several atomic distances in our case), the classical scaling assumption neglecting interaction of the boundary layers needs to be reconsidered.

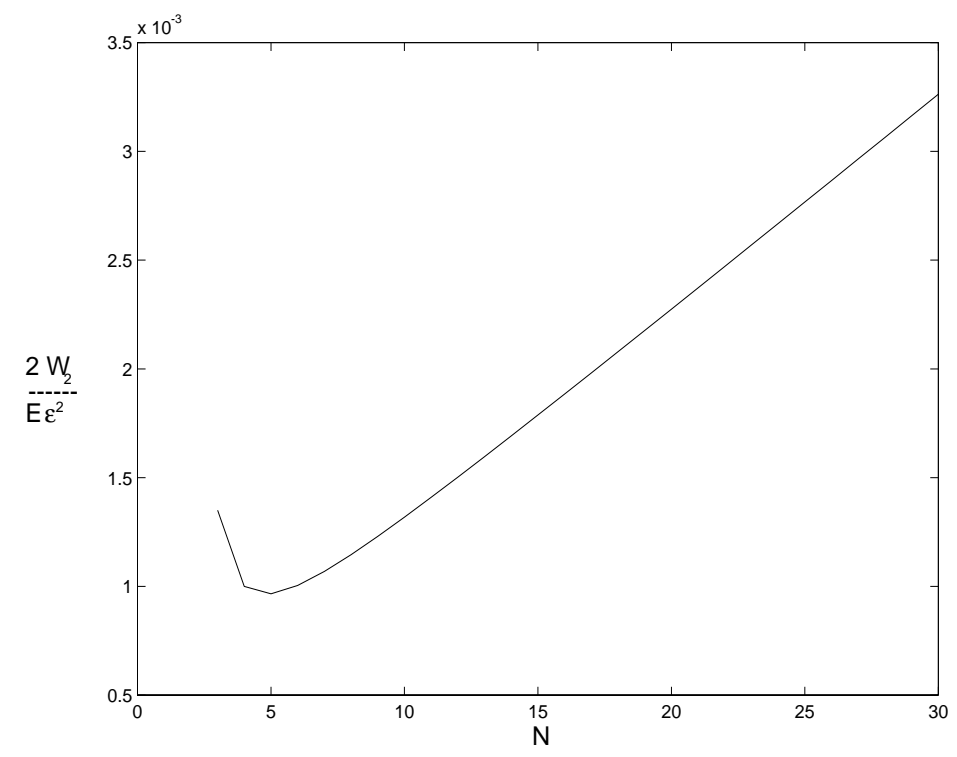

Fig. 11 The elastic part of energy of a chain as a function of the number of particles. Here $\mu=-2, B_{0}=10^{-4}$.

The loading device (9.4) is not the only non-local generalization of what is conventionally called hard device in the local theories. Thus, one can consider a special mixed device with the imposed overall strain $\bar{\varepsilon}$ and zero "long range" forces $f_{1}$ and $f_{2}$

$$
u_{N}=-u_{o}=\frac{N}{2} B_{o} \bar{\varepsilon}, \quad f_{1}=f_{2}=0
$$

For the boundary conditions (9.10) the constants $B^{\prime}, C^{\prime}, D^{\prime}$. in Case I take the form (see [C00] for details)

$$
B^{\prime}=\frac{N B_{o} \bar{\varepsilon} \cosh \frac{N \lambda_{1}}{2} \sinh \lambda_{1}}{N \cosh \frac{N \lambda_{1}}{2} \sinh \lambda_{1}-2 \sinh \frac{N \lambda_{1}}{2}}
$$




$$
\begin{gathered}
D^{\prime}=-\frac{N B_{o} \bar{\varepsilon}}{N \cosh \frac{N \lambda_{1}}{2} \sinh \lambda_{1}-2 \sinh \frac{N \lambda_{1}}{2}} \\
C^{\prime}=0
\end{gathered}
$$

After tedious but straightforward calculations we obtain the expression

$$
W_{2}(\bar{\varepsilon})=N B_{0} \frac{E \bar{\varepsilon}^{2}}{2}\left(1+\frac{2 \tanh \frac{N \lambda_{1}}{2}}{N \sinh \lambda_{1}-2 \tanh \frac{N \lambda_{1}}{2}}\right)
$$

Notice that (9.14) has the same limit as (9.8) at large $N$ but behaves differently at small $N$. These non-Saint Venant's deviations which we study in more details in the next section are characteristic of the theories exhibiting size effect.

\section{Elastic moduli}

To illustrate the difference between the loading devices (9.4) and (9.10) in this section, we compute the dependence of the corresponding effective overall elastic moduli of the chain on the number of particles. To define the modulus, we first introduce the elastic energy density

$$
w_{2}(\bar{\varepsilon})=\frac{W_{2}(\bar{\varepsilon})}{N B_{0}} .
$$

Then, the elastic modulus can be defined as

$$
\bar{E}=\frac{d^{2} w_{2}}{d \bar{\varepsilon}^{2}}=(\alpha+4 \gamma) \frac{d B^{\prime}}{d \bar{\varepsilon}}
$$

The final expression can be written in the form

$$
\bar{E}=E(1+\delta(N))
$$

Here $E$ is given by (9.9) and correction factor $\delta(N)$ depends on the specifics of the loading device disappearing in the limit $N \rightarrow \infty$. It the case of the hard device (9.4) and $\mu<-1$, we obtain 


$$
\delta_{1}(N)=\frac{\tanh \frac{N \lambda_{1}}{2}}{[N(\mu+1)-1] \tanh \frac{N \lambda_{1}}{2}+N \sqrt{\mu(\mu+1)}} .
$$

Similarly, for the hard device (9.10), the computations give

$$
\delta_{2}(N)=\frac{\tanh \frac{N \lambda_{1}}{2}}{N \sqrt{\mu(\mu+1)}-\tanh \frac{N \lambda_{1}}{2}} ;
$$

The behavior of the function $\delta_{1}(N)$ and $\delta_{2}(N)$ is illustrated in Fig.12. At small $N$ we again observe a characteristic size effect: the dependence of overall elastic modulus on the length of the chain. We notice that the qualitative behavior of the functions $\delta_{1}(N)$ and $\delta_{2}(N)$ is similar with both expressions overestimating at small $N$ the value of the elastic modulus for the infinite chain.

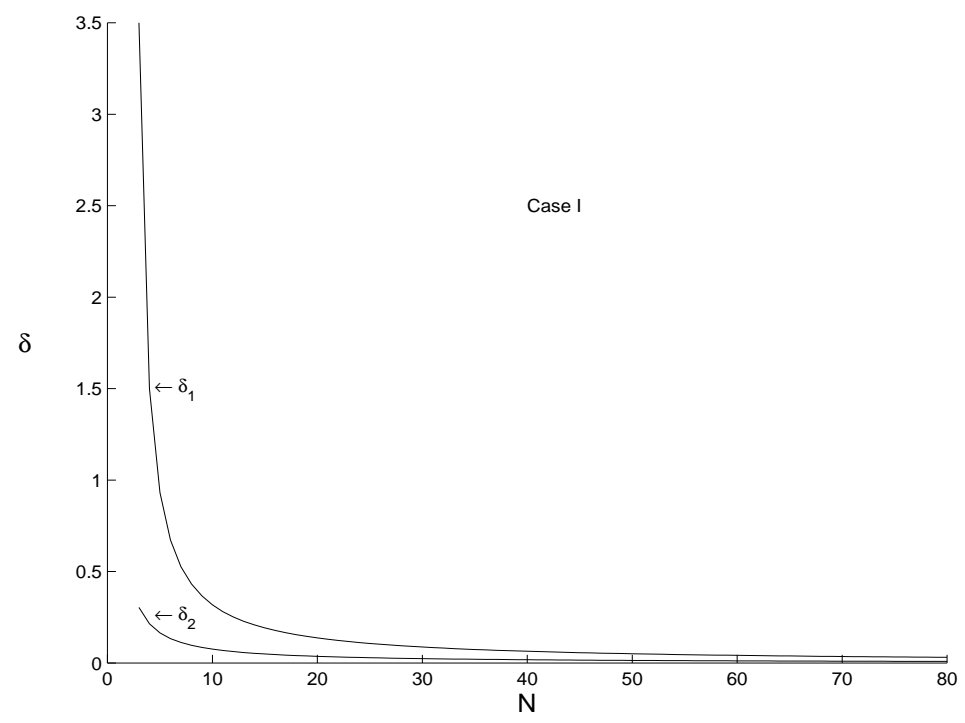

Fig.12. Behavior of the function $\delta_{1}(N)$ and $\delta_{2}(N)$ in the Case I (with $\mu=-2$ ).

The fact that the modulus gets higher as the specimen thickness tends to zero is in agreement with the findings of [W92] and seems to be supported by the experimental observations (e.g. [L99]). The results of this section suggest that the elasticity of the ultra-thin objects may deviate substantially from the bulk elasticity of the material. This is a natural consequence of the fact that at the atomic sizes surface effects dominate bulk properties. 


\section{Concluding remarks}

In this paper we constructed a complete set of static equilibrium solutions for a finite discrete chain with generic linear interactions of both nearest and next to nearest neighbors. The behavior of a linear chain with the interaction of the nearest neighbors only is trivial: the particles are always equidistant. By introducing the NNN interaction, we were able to capture some of the non-locality of the non-one-dimensional discrete models. The main focus, however, was on the effects of the hyper-pre-stress. Hyper-prestress appears in the model if $\mathrm{NN}$ and $\mathrm{NNN}$ springs have incompatible reference lengths. In this case the two interactions, favoring different spacings compete, producing configurations, which are internally stressed even in the absence of the applied forces.

The two main effects of the nonzero hyper-pre-stress are the surface relaxations and the internal modulations, which may be commensurate or non-commensurate with the reference lattice. Contrary to most of the previous work, our emphasis was on a description of a finite chain with the interacting boundaries. In particular, we studied effects of non-local loading and gave a detailed solution of the equilibrium problem for the case of a generic soft device. As a part of the solution, we singled out combinations of applied forces responsible for bulk deformation and specified self-equilibrated force systems contributing to the boundary layers only.

When the chain contains sufficiently large number of particles, the boundary layers around the free surfaces become autonomous and one can define the corresponding excess energy. Our model allows one to compute this energy explicitly and to separate the conventional contribution, due to the background cohesion from the contribution due to the hyper-pre-stress. We show, that the cohesive part of the surface energy is always positive, while the hyper-pre-stress related contribution may have different signs depending on the ratio of elastic moduli characterizing $\mathrm{NN}$ and $\mathrm{NNN}$ interactions. One of the important conclusions is that for an object with the size of the order of the internal length scale, the effective surface energy can no longer be considered independent of the size and the shape of the body; one can expect the internal length to be on the order of 10 lattice spacings. Our analysis of the size dependence of the elastic modulus suggests, that due to the interaction of the boundary layers, the ultra-thin bodies will exhibit anomalous stiffness, in tension, torsion and bending.

One important question, which could not be fully addressed in harmonic approximation concerns with the stability of the equilibrium configurations. Previous analyses of the linear stability for the infinite chain with $\mathrm{NNN}$ interactions have lead to the well known instability conditions $\{\alpha<0, \alpha+4 \gamma<0\}$ (e.g. [GW62, 65, K82]). It is not hard to see that the application of these inequalities results in the instability of all configurations associated with our Case II $(-1<\mu<0)$. For a finite chain, the computations presented in the Appendix show that the domain of instability is strictly inside the above intervals which means that some of the Case II solutions are stable (the ones with $\left.\alpha-4 \gamma \min _{q} \mu_{c}(q)>0, \alpha-4 \gamma \max \mu_{c}(q)>0\right)$. On the other hand since the instability can not be judged based on the linear part of the model only, the precise conditions of stability will depend on the nonlinear terms neglected in the present study. In fact, by adding to our energy in Case II quartic terms (guaranteeing sufficient growth of the energy at infinity), one can obtain stable periodic microstructures even in the 
infinite domain (e.g. [J88]). Stable two-dimensional quasi-periodic microstructures in the infinite nonlinear lattices have also been studied in the literature (see for instance [CMV96]).

Another interesting question, which has not been addressed in this paper is related to the derivation of an adequate continuum approximation for the NNN model with the hyper-pre-stress. The standard long wave approximation will work only in the range of parameters where the boundary layers are sufficiently wide and the oscillations on the scale of the lattice are absent; this means our Case I with $\mu$ close to -1 . The main difficulty arises from the necessity of adding to the bulk energy appropriate nullLagrangians responsible for the formation of the boundary layers. This issue deserves a separate analysis and will be considered elsewhere.

Our results may have some bearing on the criteria of failure in solids. Thus we show that when a chain with a hyper-pre-stress is being broken, two quite different phenomena are taking place simultaneously. First, the boundary layers are created with the corresponding energy expenditure solely due to the hyper-pre-stress. Second, the two freshly formed surfaces need to be separated and now the corresponding work has basically nothing to do with the hyper-pre-stress. The above two-stage scenario suggests an idea that the dependence of the surface energy on the separation of the crack surfaces may be bi-modal with two plateaus: the smaller one corresponding to the energy of the boundary layers and bigger one corresponding to the energy of the ultimate de-cohesion. This idea, formulated as an assumption of the non-concavity of the surface energy, has been recently used in [DT01] to simulate fractured configurations where several microcracks (or pre-cracks) coexist with a developed macro-crack.

In the context of fracture mechanics, it is also of interest to study directly the behavior of a discrete model with the nonlinear interaction of the Lennard-Jones type: $w_{1}(z)=w_{2}(z)=w(z)$. For close to homogeneous equilibrium configurations, our linear analysis can provide a good approximation to the nonlinear solution. To insure the agreement between the two models, the parameters of the harmonic approximation $\alpha, \gamma$, $\xi_{1}, \xi_{2}, w_{1}{ }^{0}, w_{2}{ }^{0}$ must be chosen compatible with the nonlinear potential $w(z)$. By cutting a chain sufficiently far away from the external surfaces one can see that the quasihomogeneous particle spacing $B$ can be found from the equation $w^{\prime}(B)+2 w^{\prime}(B)=f$, where $f=f_{1}+f_{2}=f_{3}+f_{4}$ (cf. 2.13). Then by linearizing the nonlinear potential around the homogeneous states with the spacings $B(f)$ and $2 B(f)$, one obtains the following parameters of the "tangential" model

$$
\begin{array}{cc}
w_{1}^{0}=w(B)-\frac{w^{\prime 2}(B)}{2 w^{\prime \prime}(B)}, & w_{2}^{0}=w(2 a)-\frac{w^{\prime 2}(2 B)}{2 w^{\prime \prime}(2 B)}, \\
\xi_{1}=a-\frac{w^{\prime}(B)}{w^{\prime \prime}(B)}, & \xi_{2}=a-\frac{w^{\prime}(2 B)}{2 w^{\prime \prime}(2 B)}, \\
\alpha=w^{\prime \prime}(B), & \gamma=w^{\prime \prime}(2 B) .
\end{array}
$$

In particular the non-dimensional parameter 


$$
\mu=\frac{w^{\prime \prime}(B)}{4 w^{\prime \prime}(2 B)}
$$

can clearly be in any of the three generic domains. Notice also that in the present setting, the hyper-pre-stress $\xi$ is generically different from zero

$$
\xi=\frac{w^{\prime}(B)}{2 w^{\prime \prime}(B)}-\frac{w^{\prime}(2 B)}{4 w^{\prime \prime}(2 B)} .
$$

The linear approximation obviously fails when one approaches the bifurcation points (4.14) indicating the onset of instability. The analysis of the associate nonlinear model can reveal the structure of the bifurcated branches leading to fractured lattice configurations. Partial theoretical results concerning the behavior of an infinite NNN system with Lennard-Jones potential can be found in [BG00]; selected numerical computations for a finite chain were reported in [TB96]. In spite of these efforts and the fact that the associated NN problem is thoroughly studied (e.g. [T96, BGD99]), the general bifurcational diagram for the Lennard-Jones NNN problem is far from being known.

In a slightly different but related context of the discrete theory of phase transitions, the introduction of the NNN interaction has been shown to eliminate the degeneracy of the simpler NN model through effective introduction of the interface energy (e.g. [RT97, PT00]). The origin of this interface energy is the structural relaxation around the internal surfaces (phase or twin boundaries). Based on the analogy with the present work, one can speculate that at sufficiently small scales the effective interface energy will strongly depend on the separation of the interfaces. This may explain, for example, why nano-scale particles of smart materials do not exhibit a characteristic microstructure.

\section{Acknowledgements}

This work was supported by the NSF grant DMS-9803572 (LT) and by the Graduate Fellowship Grant from the French Government (MC). The authors would like to thank J.J. Marigo and the reviewers for helpful suggestions and each other's Institutions for the hospitality provided during much of the work.

\section{References}

[BDG99] Braides A., Del Maso G., Garroni A. 1999 "Variational formulation of softening phenomena in fracture mechanics: the one-dimensional case”, Arch. Rational Mech. Anal. ,146, 23-58

[BG99] Braides A., Gelli M. 1999 "Limits of discrete systems with long range interactions" Preprint SISSA 
[C63] Casal P. 1963 "Capillarite interne en mechanique” C.R.Acad. Sci. 256, 380-3822

[CMV96] Chow S.N., Mallet-Paret J., Van Vleck E.S. 1996 "Pattern formation and spatial chaos in spatially discrete evolution equations", Random and Comp. Dynamics.. ,4, 109-178

[DT01] Del Piero G., Truskinovsky L., 2001 "Macro and micro cracking in one-dimensional elasticity", Int. J. Solids Struct., 38, 1135-1148

[E92] Eringen A. 1992 "Vistas of nonlocal continuum physics” Int. J. Eng. Sci. 30, 1551-1565

[FM96] Fosdick R. Mason D. 1996 "Single phase energy minimizers for materials with nonlocal spatial dependence" Quart. Appl. Mat. 54, 161-195

[GW65] Gazis D., Wallis R. 1965 "Surface tension and surface modes in semi-infinite lattices" Surface Science, 3, 19-32

[GMH61] Germer L., MacRae A., Hartman C. J. 1961 “(100) Nickel Surface “ J. Appl. Phys., 32 24322439

[HLS92] Houchmandzadeh B., Lajzerowicz J., Salje E. 1992 "Relaxations near surfaces for first-,secondand third-neighbour interactions: theory and applications to polytypism". J. Phys. Cond. Matter 4 9779-9794.

[JT82] Janssen T., Tjon J. 1982 "Microscopic model for incommensurate crystal phases", Phys Rev. B 25, 3767-3785

[K65] Kunin I. 1965 "Elastic media with microstructure - I (one-dimensional models)", Springer

[L95] Lakes R. 1995 "Experimental methods for study of Cosserat elastic solids and other generalized continua", in: Continuum models for materials with Microstructure, ed. H.B. Muhlhaus, John Willey Ltd.,1-25

[LSD99] Lee W., Salje E. , Dove M. 1999 "Effect of surface relaxations on the equilibrium growth morphology of crystal: platelet formation". J. Phys. Cond. Matter. 11, 7385-7410

[LL59] Levy H., Lessman F.1959 "Finite difference equations”. Pitman

[M65] Mindlin R..1965 "Second gradient of strain and surface tension in linear elasticity". Int. J. Solids Structures, 1, .417-438.

[MPT98] Mizel V.L., Peletier L.A., Troy W.C. 1998 "Periodic phases in second order materials", Arch. Rational Mech. Anal. ,145, 343-382

[P91] Pouget J. 1991 "Dynamics of patterns in ferroelastic-martensitic transformations I. Lattice model", Phys. Rev. B 43, 3575-3581

[PT00] Puglisi, G.; Truskinovsky, L. 2000 "Mechanics of a discrete chain with bi-stable elements". $J$. Mech. Phys. Solids $\mathbf{4 8}, 1--27$.

[RT01] Ren X., Truskinovsky L. 2001 "Finite scale microstructures in nonlocal elasticity", J. Elast, ???, ???-???

[RT97] Rogers R., Truskinovsky L. 1997 “Discretization and hysteresis”, Physica B, 233, 370-375

[T64] Toupin R. 1964 “Theories of elasticity with couple stress" .Arch Rational Mech. Anal. 17, 7-112 
[TG64] Toupin R.A. and Gazis D.C. 1964 "Surface effects and initial stress in continuum and lattice models of elastic crystals". In: Proceedings of the International Conference on Lattice Dynamics, ed. Wallis R.F., 597-605.

[TB93] Triantafillydis N. and Bardenhagen S.1993 "On higher order gradient continuum theories in 1-D nonlinear elasticity. Derivation from and comparison to the corresponding discrete models". $J$. Elasticity, 33, 259-293

[T96] Truskinovsky L.1996 "Fracture as a phase transformatio", in: Contemporary research in mechanics and mathematics of materials, ed. R. Batra and M. Beatty, CIMNE, Barselona,.322-332

[VS95] Vardoulakis I. Sulem J. 1995 Bifurcation analysis in Geomechanics, Chapman \& Hall

[W92] Wu C. 1992 "Cohesive elasticity and surface phenomena” Quart. Appl. Mat. 50, 73-100

\section{Appendix}

The equilibrium configurations of a chain in a soft device, studied in the main body of the paper, satisfy the equilibrium and boundary conditions $\partial P / \partial x_{i}=0$, where $P$ is the total energy of the system (see Sections 3,4$)$. To study the linear stability of the equilibrium solutions one needs to analyze the positive definiteness of the corresponding Hessian matrix $\partial^{2} P / \partial x_{i} \partial x_{j}$. In terms of the moduli $\alpha$ and $\gamma$ this $(N+1) \times(N+1)$ matrix takes the form

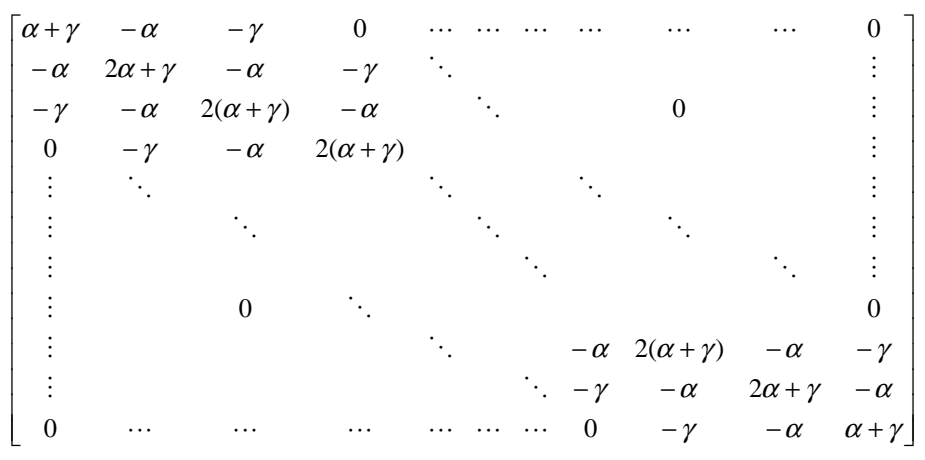

The principal minors of this matrix satisfy the following equations (see a related case in [WG65])

$$
\begin{aligned}
& \Delta_{1}=\alpha+\gamma \\
& \Delta_{2}=(\alpha+2 \gamma)(\alpha+\gamma)-\gamma^{2} \\
& \Delta_{k}=(\alpha+2 \gamma) \Delta_{k-1}-\gamma^{2} \Delta_{k-2} \quad, \quad 3 \leq k \leq N-1 \\
& \Delta_{N}=(\alpha+\gamma) \Delta_{N-1}-\gamma^{2} \Delta_{N-2} \\
& \Delta_{N+1}=0
\end{aligned}
$$

It is not hard to see that the first two equations provide "initial conditions" for the main recurrent relation for $\Delta_{k}$; the value of $\Delta_{N}$ can be computed after all other principal minors are known. The fact that the minor of rank $N+1$ is equal to zero can be linked to the translational invariance of the chain in the soft device (and related arbitrariness of the constant $A$ from Section 3). 
A general solution of the main difference relation for the minors can be written as a combination of the monomials $z^{k}$, with $z$ being a root of the following characteristic equation

$$
z^{2}-(\alpha+2 \gamma) z+\gamma^{2}=0
$$

If $\gamma \neq 0$ (NNN interactions are present) the two roots of the characteristic equations can be written in the form $z_{1,2}=\gamma \rho_{3,4}$, with $\rho_{3,4}$ given by (3.6). Now, by substituting the "initial data" for the minors of rank one and two into the general solution one obtains the following explicit relations

- $\quad-\infty<\mu<-1$ (Case I)

$$
\begin{aligned}
& \Delta_{k}=(-\gamma)^{k} \frac{\sinh \left[(k+1 / 2) \lambda_{1}\right]}{\sinh \left(\lambda_{1} / 2\right)}, \quad 1 \leq k \leq N-1 \\
& \Delta_{N}=2(-\gamma)^{N} \operatorname{coth}\left(\lambda_{1} / 2\right) \sinh \left(N \lambda_{1}\right)
\end{aligned}
$$

- $\quad-1<\mu<0$ (Case II)

$$
\begin{aligned}
& \Delta_{k}=(-\gamma)^{k} \frac{\sin \left[(k+1 / 2) \lambda_{2}\right]}{\sin \left(\lambda_{2} / 2\right)} \quad, \quad 1 \leq k \leq N-1 \\
& \Delta_{N}=2(-\gamma)^{N} \cot \left(\lambda_{2} / 2\right) \sin \left(N \lambda_{2}\right)
\end{aligned}
$$

- $\quad 0<\mu<+\infty$ (Case III)

$$
\begin{aligned}
& \Delta_{k}=\gamma^{k} \frac{\cosh \left[(k+1 / 2) \lambda_{1}\right]}{\cosh \left(\lambda_{1} / 2\right)} \quad, \quad 1 \leq k \leq N-1 \\
& \Delta_{N}=2 \gamma^{N} \tanh \left(\lambda_{1} / 2\right) \sinh \left(N \lambda_{1}\right)
\end{aligned}
$$

Here parameters $\lambda_{1}>0$ and $\lambda_{2} \in(0, \pi)$ are the same as in Section 3. From these expressions for the principal minors we observe that the stability conditions depend on both material parameters $\alpha$ and $\gamma$; we recall that the equilibrium configurations depend only on their non-dimensional ratio $\mu$.

The analysis of Cases I and III is rather straightforward. Thus, in Case I all minors are positive if and only if $\gamma<0$. This generates stability domain $\{\gamma<0, \alpha+4 \gamma>0\}$ and instability domain $\{\gamma>0$, $\alpha+4 \gamma<0\}$. Similarly, in Case III all minors are positive if and only if $\gamma<0$. This produces $\{\gamma>0$, $\alpha>0\}$ as stability domain and $\{\gamma<0, \alpha<0\}$ as instability domain. The situation is more subtle in Case II, which, for infinite chains turns out to be completely unstable.

In Case II one has to consider two possibilities: $\gamma>0$ and $\gamma<0$. If $\gamma>0$ the principal minors are positive if and only if

$$
\begin{aligned}
& \frac{2 q_{k}-k}{k+1 / 2} \pi \leq \lambda_{2} \leq \frac{2 q_{k}+1-k}{k+1 / 2} \pi \quad, \quad 1 \leq k \leq N-1 \\
& \frac{2 q_{N}-N}{N} \pi \leq \lambda_{2} \leq \frac{2 q_{N}+1-N}{N} \pi
\end{aligned}
$$

Here for every $1 \leq k \leq N$, parameters $q_{k}$ span all positive integers compatible with the constraint $\lambda_{2} \in(0, \pi)$. One can show that the above admissible intervals are incompatible which means that the corresponding 
equilibrium states are unstable. As a result we obtain another instability domain $\{\alpha+4 \gamma>0, \alpha<0\}$. If $\gamma<0$, the principal minors are positive if and only if

$$
\begin{aligned}
& \frac{2 q_{k}}{k+1 / 2} \pi \leq \lambda_{2} \leq \frac{2 q_{k}+1}{k+1 / 2} \pi \quad, \quad 1 \leq k \leq N-1 \\
& \frac{2 q_{N}}{N} \pi \leq \lambda_{2} \leq \frac{2 q_{N}+1}{N} \pi
\end{aligned}
$$

where again for each $1 \leq k \leq N$ the positive integers $q_{k}$ must be compatible with the constraint $\lambda_{2} \in(0, \pi)$. The largest admissible domain in this case is: $0<\lambda_{2}<\pi / N$ which in terms of $\mu$ is equivalent to the following stability conditions

$$
-1<\mu<-\frac{1}{2}\left[1+\cos \left(\frac{\pi}{N}\right)\right]=\min _{q} \mu_{c}(q)
$$

Here the function $\mu_{c}(q)$ is given by (4.14). By rewriting above conditions in terms of $\alpha$ and $\gamma$ we obtain $\left\{\alpha-4 \gamma \min _{q} \mu_{c}(q)>0, \alpha>0\right\}$ as a stability domain and $\left\{\alpha-4 \gamma \min _{q} \mu_{c}(q)<0, \alpha>0\right\}$ as an instability domain.

The complete characterization of the stability domains requires consideration of the limiting cases $\mu=-1$ and $\mu=0$. In the first case $(\mu=-1)$ we obtain

$$
\begin{aligned}
& \Delta_{k}=(-\gamma)^{k}(2 k+1) \quad, \quad 1 \leq k \leq N-1, \\
& \Delta_{N}=4(-\gamma)^{N} N
\end{aligned}
$$

which means that for stability it is necessary to have $\gamma<0$. In the second case $(\mu=0)$, we get

$$
\begin{aligned}
& \Delta_{k}=(-\gamma)^{k} \quad, \quad 1 \leq k \leq N-1 \\
& \Delta_{N}=0
\end{aligned}
$$

meaning instability if $\gamma>0$ and neutral stability if $\gamma<0$. The case $\gamma=0$ (NN chain) has to be treated separately; in this case $\Delta_{k}=\alpha^{k}$ for $1 \leq k \leq N$ and stability requires $\alpha>0$. of stability

By collecting all stability intervals indicated above, we obtain the following combined conditions

$$
\left\{\alpha-4 \gamma \min _{q} \mu_{c}(q)>0, \gamma<0\right\} \text { and }\{\alpha>0, \gamma>0\}
$$

If we now recall that $\max _{q} \mu_{c}(q)=0$, we can rewrite these conditions in the following form

$$
\left\{\alpha-4 \gamma \min \mu_{c}>0, \alpha-4 \gamma \max \mu_{c}>0\right\} \text {. }
$$

We remark that in particular this means that the chain is unstable for $\min _{q} \mu_{c}(q)<\mu<\max _{q} \mu_{c}(q)$.

Conditions $(*)$ can be compared to the corresponding conditions for the infinite chain

$$
\{\alpha+4 \gamma>0, \quad \alpha>0\}
$$


Since $-1 \leq \min _{q} \mu_{c}(q)<\max _{q} \mu_{c}(q) \leq 0$, one can see that domain $(*)$ is larger than domain $(* *)$, which reflects the obvious fact that a finite subsystem is more stable than the whole infinite system. By using the explicit relation for $\mu_{c}(q)$ (4.14) we can compute stability limits for the chain of arbitrary length. In particular, for the shortest NNN chain with $N=3$, conditions of stability (*) reduce to $\alpha+3 \gamma>0, \alpha>0$. The substantial stretching of the stability domain in this case comparing to (**) illustrates the enhanced stability of ultra-thin objects.

It is not hard to see that due to the broadening of the class of admissible variations, the stability conditions for the soft device will also be sufficient for the cases of hard and mixed devices. What is more interesting, conditions (*) are also sufficient for those devices if parameters $\mu_{\mathrm{c}}$ are taken to be bifurcational points associated with the hard or mixed devices, accordingly. The fact that $\min _{q} \mu_{c}(q)$ and $\max _{q} \mu_{c}(q)$ give the lower and upper boundaries for the domain of instability in the $\mu$-space follows from the observation that in both points the minimal eigenvalue of the Hessian matrix becomes negative. To prove that $(*)$ represent exact stability conditions in those cases one needs to use the fact that additional constraints can only increase the value of the minimal eigenvalue and that its dependence on elastic moduli $\alpha$ and $\gamma$ is continuous and monotone. All these statements can be checked without difficulty. 